\title{
Bayesian analysis of quark spectral properties from the Dyson-Schwinger equation
}

\author{
Christian S. Fischer, ${ }^{1,2}$ Jan M. Pawlowski, ${ }^{3,4}$ Alexander Rothkopf, ${ }^{5}$ and Christian A. Welzbacher ${ }^{1}$ \\ ${ }^{1}$ Institut für Theoretische Physik, Justus-Liebig Universität Gießen, 35392 Gießen, Germany \\ ${ }^{2}$ HICforFAIR-Gießen \\ ${ }^{3}$ Institut für Theoretische Physik, Universität Heidelberg, \\ Philosophenweg 16, 69120 Heidelberg, Germany \\ ${ }^{4}$ ExtreMe Matter Institute EMMI, GSI Helmholtzzentrum für Schwerionenforschung mbH, \\ 64291 Darmstadt, Germany \\ ${ }^{5}$ Institut für Theoretische Physik, Universität Heidelberg, \\ Philosophenweg 12, 69120 Heidelberg, Germany
}

(Received 7 August 2017; published 6 July 2018)

\begin{abstract}
We report results on the quark spectral function in the Landau gauge at finite temperature, determined from its Dyson-Schwinger equation. Compared to earlier quenched results, this study encompasses unquenched $N_{f}=2+1$ fermion flavors in the medium. For the computation of real-time spectra, we deploy the recent Bayesian Reconstruction (BR) method and develop a new prior in order to better assess the inherent systematic uncertainties. We identify the quark quasiparticle spectrum and analyze the (non) appearance of zero modes at or around the pseudocritical temperature. In both, the fully unquenched system and a simpler truncation, using a model for the gluon propagator, we observe a characteristic two-peak structure at zero three-momentum. The temperature dependence of these structures, in case of the gluon propagator model, is different than observed in previous studies. For the back-coupled and unquenched case, we find interesting modifications at and around the pseudocritical transition temperature.
\end{abstract}

DOI: 10.1103/PhysRevD.98.014009

\section{INTRODUCTION}

The wealth of data produced in heavy ion collision experiments at the Relativistic Heavy-Ion Collider and the LHC has lead to interesting insights about the nature of the quark-gluon plasma (QGP) in various temperature regimes (see e.g., Refs. [1-5] and references therein). Thermal and transport properties of the QGP are encoded in the correlation functions of QCD. In particular, they can be assessed from real-time properties of QCD's most basic correlation functions, the quark [6-14] and gluon propagators [15-19]. A prominent example is the dilepton production in a heavy ion collision. It can been related to the spectral properties of thermalized quasiparticles and specifically to the dispersion relation of quarks [20-24]. Another important example is QCD transport coefficients, that have been expressed in terms of single particle spectral functions of the fundamental fields, the quarks and gluons $[16,17]$. In summary, a detailed understanding of a potential

Published by the American Physical Society under the terms of the Creative Commons Attribution 4.0 International license. Further distribution of this work must maintain attribution to the author(s) and the published article's title, journal citation, and DOI. Funded by SCOAP ${ }^{3}$. quasiparticle spectrum in the QGP, in particular close to the chiral phase transition, is highly desirable.

In this work, we focus on the quark spectral function encoding the quark dispersion relation and decay width in the medium. At large temperatures, reliable results have been obtained in the hard-thermal loop (HTL) expansion [25-27]. In this regime, the quark spectral function shows two excitations in the dispersion relation, the ordinary quark with a positive ratio of chirality to helicity and a collective "plasmino" mode with a corresponding negative ratio. Both have thermal masses of the order $g T$ and decay widths of the order $g^{2} T$, where $g$ is the coupling constant and $T$ is the temperature. The two excitations are accompanied by a continuum contribution from a branch cut in the quark propagator due to Landau damping, i.e., the absorption of a spacelike quark by a hard gluon or hard antiquark.

Beyond systematic weak-coupling expansions, there is no straightforward approach for the determination of the spectral function. Model calculations offer qualitative insights [8,28-30] which, however, need to be corroborated in more fundamental approaches. Models for quark spectral functions constructed along the lines of the HTL results have been fitted to data from quenched lattice QCD [10,11] and quenched Dyson-Schwinger calculations [6]. Again, such an approach offers qualitative insights but suffers from 
potential biases involved in the model building. This holds in particular for temperatures around the (pseudo)critical one, where HTL is not expected to be reliable.

In principle, functional approaches like Dyson-Schwinger equations (DSE) and the functional renormalization group offer the possibility to determine the two-point correlators in the complex momentum plane, thus allowing for a direct extraction of the corresponding spectral function. This has been performed successfully for the gluon propagator at zero temperature in Ref. [15]. At finite temperatures, direct computations in the complex frequency plane have been carried out in matter systems; see e.g., Refs. [31-34]. However, the additional conceptual and numerical challenges have delayed similar direct analyses in QCD so far.

There are, however, approaches that allow us to extract spectral functions from numerical data in the spatial Euclidean momentum region. These approaches utilize the fact that the spectrum is related to the Euclidean correlator via an integral transform, which needs to be inverted. This is a classic ill-posed inverse problem, and Bayesian inference can be used to give meaning to it, by systematically incorporating additional prior information available. Among the different implementations of the Bayesian reconstruction strategy is the popular Maximum Entropy Method (MEM) [35-37], which originates in two-dimensional image reconstruction. It has been deployed for the study of quarks in cold and dense matter [7] and quarks at and around the (pseudo)critical temperature [12-14]. A method similar in spirit as the MEM but instead using a quadratic regulator has been applied to study gluon spectra in Ref. [18].

As has been discussed e.g., in Ref. [38], the MEM based approaches have to deal with several issues. The main difficulty is that of flat directions in the regulator functional in case of positive definite spectra. In practice, this leads to very slow convergence in case that a large number of data points is supplied. The second point is related to the weighting of data and prior information, which conventionally is implemented via computing the so-called evidence probability distribution. This step relies on a Gaussian approximation, which in practice is difficult to justify; see also Appendix C.

In order to overcome these and further difficulties, a novel implementation of the Bayesian strategy has been recently developed [38]. It is specifically designed for the solution of one-dimensional inverse problems. Its generalization to arbitrary spectra [39] has been applied for extracting spectral properties of gluons at finite temperature [19] in lattice QCD. In this study, we both deploy the original BR method and develop in addition a new "lowringing" BR-type prior functional, which allows us to unambiguously distinguish between peaked structures present in the underlying correlator data and numerical ringing artifacts (see e.g., the discussion in Ref. [40]) common to inverse problems (cf. the Gibbs phenomenon).
We apply our new method to temperature dependent quark propagators obtained from two different truncation schemes for the quark and gluon DSEs. On the one hand, we reanalyze a model truncation for the gluon propagator and compare to previous results [12-14]. In these works, a frequency zero mode in the spectral function at zero spatial momentum has been identified in addition to the two conventional symmetric peaks at finite frequency. Due to the symmetry of the spectral function, we will nevertheless refer to such a constellation of spectral features in the following as a two-peak structure and plot spectra only in the positive frequency regime.

Since a zero mode does not appear in HTL studies, it has been attributed to the strong interaction physics governing the transition around the (pseudo)critical temperature and signaling the formation of the quark-gluon plasma. While the appearance of this low frequency structure has been found to be robust under variations within a class of truncation schemes using models for the gluon [14], it remains to be seen whether this is also true for the fully unquenched system. In this article, we therefore study in addition a truncation based on Refs. $[6,41]$ to include results for the unquenched quark propagator with $N_{f}=2+1$. This truncation offers direct control over the Yang-Mills sector by explicitly taking quark loop effects in the gluon propagator into account. The resulting prediction for the unquenched gluon propagator at finite temperature [41] has been shown to agree with corresponding lattice results of Ref. [42]. Furthermore, the temperature dependence of the chiral condensate evaluated on the lattice [43] has been reproduced. We therefore may expect realistic and quantitative results for the spectral functions as well.

The article is organized as follows. In the next section, we summarize the framework to determine the quark propagator at finite temperature and chemical potential. Since all technical details have been given elsewhere, see Refs. [12-14] for the model gluon and Refs. [41,44-46] for the unquenched system, we remain very brief. Section III is devoted to the Bayesian BR method reconstruction and the specific improvements we use in this work. Our results for the quark spectral functions are presented and discussed in Sec. IV. We conclude in Sec. V.

\section{QUARK PROPAGATOR AT FINITE TEMPERATURE AND CHEMICAL POTENTIAL}

\section{A. Quark Dyson-Schwinger equation}

In order to determine the quark propagator at finite temperature and chemical potential, we work with the Euclidean metric version of QCD and use the Matsubara formalism. The renormalized quark Dyson-Schwinger equation is then given by

$$
S^{-1}\left(i \omega_{p}, \boldsymbol{p}\right)=Z_{2} S_{0}^{-1}\left(i \omega_{p}, \boldsymbol{p}\right)+\Sigma\left(i \omega_{p}, \boldsymbol{p}\right) .
$$




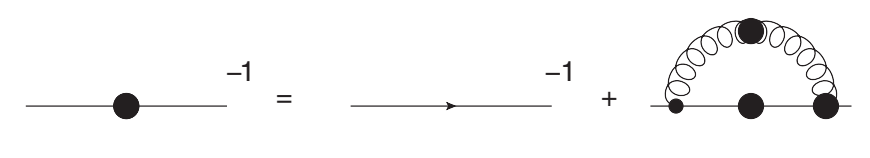

FIG. 1. The quark Dyson-Schwinger equation. Dressed propagators and vertices are denoted by large filled dots.

Here, the inverse full quark propagator is denoted by $S^{-1}\left(i \omega_{p}, \boldsymbol{p}\right)$, and its inverse bare counterpart is denoted by $S_{0}^{-1}\left(i \omega_{p}, \boldsymbol{p}\right)$. We follow the conventions of Ref. [6] and explicitly use imaginary arguments for the energy in all functions. The dependence of all functions on the renormalization point is left implicit, and $Z_{2}$ denotes the wave function renormalization constant of the quark. The quark Matsubara frequencies are given by $\omega_{p}=\left(2 n_{p}+1\right) \pi T$ with temperature $T$. The Dirac structure of the inverse propagators at finite $\mathrm{T}$ and baryochemical potential $\mu=0$ can be decomposed via

$$
\begin{aligned}
S_{0}^{-1}\left(i \omega_{p}, \boldsymbol{p}\right)= & i \gamma_{4} \omega_{p}+i \gamma \cdot \boldsymbol{p}+Z_{m} m, \\
S^{-1}\left(i \omega_{p}, \boldsymbol{p}\right)= & i \gamma_{4} \omega_{p} C\left(i \omega_{p},|\boldsymbol{p}|\right)+i \gamma \cdot \boldsymbol{p} A\left(i \omega_{p},|\boldsymbol{p}|\right) \\
& +B\left(i \omega_{p},|\boldsymbol{p}|\right) .
\end{aligned}
$$

The dressing functions $A\left(i \omega_{p},|\boldsymbol{p}|\right), B\left(i \omega_{p},|\boldsymbol{p}|\right)$ and $C\left(i \omega_{p},|\boldsymbol{p}|\right)$ carry all nontrivial information on the quarks. The quark mass renormalization constant $Z_{m}\left(\Lambda^{2}\right)$, the renormalized mass $m\left(\Lambda^{2}\right)$ and $Z_{2}\left(\Lambda^{2}\right)$ at the renormalization scale $\Lambda$ are determined within a momentum subtraction (MOM) renormalization scheme. The explicit form of the quark self-energy can be written as

$$
\begin{aligned}
\Sigma\left(i \omega_{p}, \boldsymbol{p}\right)= & \frac{16 \pi}{3} \frac{Z_{2}}{\tilde{Z}_{3}} \alpha(\Lambda) T \sum_{n_{q}} \int \frac{d^{3} q}{(2 \pi)^{3}}\left[\gamma_{\mu} S\left(i \omega_{q}, \boldsymbol{q}\right)\right. \\
& \left.\times \Gamma_{\nu}\left(i \omega_{q}, \boldsymbol{q}, i \omega_{p}, \boldsymbol{p}\right) D_{\mu \nu}\left(i \omega_{p}-i \omega_{q}, \boldsymbol{p}-\boldsymbol{q}\right)\right],
\end{aligned}
$$

with (Landau gauge) gluon propagator $D_{\mu \nu}$, quark-gluon vertex $\Gamma_{\nu}$, gauge coupling $\alpha$ and ghost renormalization constant $\tilde{Z}_{3}$.

In order to determine the quark propagator selfconsistently from its DSE (see Fig. 1), we also need to specify the dressed gluon propagator and the dressed quark-gluon vertex. The truncation used in this work has been developed over the past years $[41,44,45,47,48]$ and is guided by two main ideas. First, lattice results for the temperature dependent quenched gluon propagator are used as external input $[48,49]$ and unquenched via adding an explicit quark loop for each of the $N_{f}=2+1$ quark flavors used in this work. The resulting DSE for the gluon propagator is depicted in Fig. 2.

This approach ensures that the unquenched gluon propagator inherits the leading order temperature and chemical potential effects via the quark loops, giving a

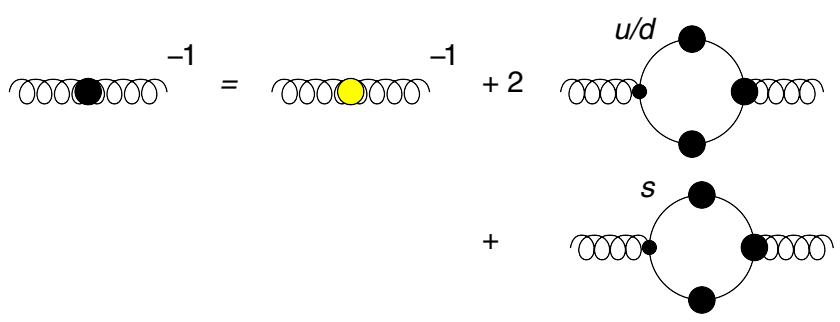

FIG. 2. The truncated gluon Dyson-Schwinger equation. The yellow dot represents input from quenched lattice QCD, whereas the dressed quark propagators in the quark loop are calculated self-consistently from the quark DSE.

contribution to the thermal mass. Second order unquenching effects in the Yang-Mills diagrams are neglected. The second element of our truncation is an approximation for the full quark-gluon vertex, which combines information from the well-known perturbative behavior at large momenta and an approximate form of the SlavnovTaylor identity at small momenta studied long ago by Ball and Chiu [50]. The explicit form of this approximate expression for the vertex is discussed in Refs. [41,45,48] and shall not be repeated here for brevity.

A much simpler system in terms of numerical effort is obtained by substituting the dressed gluon propagator in the quark DSE by a model together with a bare quark-gluon vertex. Taking into account a Debye-like mass in the longitudinal gluon, this reads $(k=q-p)$

$$
\begin{aligned}
& g^{2} D_{\mu \nu}\left(i \omega_{k}, \boldsymbol{k}\right) \Gamma_{\nu}\left(i \omega_{q}, \boldsymbol{q}, i \omega_{p}, \boldsymbol{p}\right) \\
& \quad=\left[P_{\mu \nu}^{T} D_{T}\left(\omega_{k}^{2}, \boldsymbol{k}^{2}\right)+P_{\mu \nu}^{L} D_{L}\left(\omega_{k}^{2}, \boldsymbol{k}^{2}\right)\right] \gamma_{\nu},
\end{aligned}
$$

with $P_{\mu \nu}^{T, L}$ the transverse and longitudinal projection operators with respect to the heat bath. The dressing functions are given by

$$
D_{T}=\mathcal{D}\left(\boldsymbol{k}^{2}+\omega_{k}^{2}\right), \quad D_{L}=\mathcal{D}\left(\boldsymbol{k}^{2}+\omega_{k}^{2}+m_{g}^{2}\right),
$$

where the functions

$\mathcal{D}(s)=\frac{8 \pi^{2} D}{\sigma^{4}} e^{-s / \sigma^{2}}+\frac{8 \pi^{2} \gamma_{m}}{\ln \left[\tau+\left(1+s / \Lambda_{\mathrm{QCD}}^{2}\right)^{2}\right]} \mathcal{F}(s)$

are defined with $\mathcal{F}(s)=\left[1-\exp \left(-s / 4 m_{t}^{2}\right)\right] / s, \quad \tau=$ $e^{2}-1, m_{t}=0.5 \mathrm{GeV}, \gamma_{m}=12 / 25$ and $\Lambda_{\mathrm{QCD}}=0.234 \mathrm{GeV}$. With $\sigma D=(0.8 \mathrm{GeV})^{3}$, we choose $\sigma=0.5$ as a representative value for the model parameters. This is one (the simplest) example of a class of truncation schemes that have been studied in Ref. [14] and found to agree qualitatively with each other in the resulting spectral functions for the quarks. As explained in the introduction, we use this model as a numerically easily accessible reference, which already displays an interesting two-peak structure in the resulting spectral functions. 


\section{B. Quark spectral functions and representation}

The spectral representation of the quark propagator is given by

$$
S\left(i \omega_{p}, \boldsymbol{p}\right)=\int_{-\infty}^{\infty} \frac{d \omega^{\prime}}{2 \pi} \frac{\rho\left(\omega^{\prime}, \boldsymbol{p}\right)}{i \omega_{p}-\omega^{\prime}},
$$

with spectral function $\rho(\omega, \boldsymbol{p})$ parametrized as

$$
\begin{aligned}
\rho(\omega, \boldsymbol{p})= & 2 \pi\left(\rho_{4}(\omega,|\boldsymbol{p}|) \gamma_{4}+\rho_{\mathrm{v}}(\omega,|\boldsymbol{p}|)(i \boldsymbol{\gamma} \cdot \boldsymbol{p}) /|\boldsymbol{p}|\right. \\
& \left.-\rho_{\mathrm{s}}(\omega,|\boldsymbol{p}|)\right) .
\end{aligned}
$$

Similarly to Ref. [6], we choose conventions such that the scalar dressing functions themselves agree with those introduced by corresponding lattice studies [11]. With a positive definite metric, which is not the case for gaugefixed QCD, the components of the spectral function would furthermore obey the inequality

$$
\rho_{4}(\omega,|\boldsymbol{p}|) \geq \sqrt{\rho_{\mathrm{v}}(\omega,|\boldsymbol{p}|)^{2}+\rho_{\mathrm{s}}(\omega,|\boldsymbol{p}|)^{2}} \geq 0
$$

as well as the standard sum rule

$$
Z_{2} \int_{-\infty}^{\infty} d \omega \rho_{4}(\omega,|\boldsymbol{p}|)=1,
$$

with wave function renormalization constant $Z_{2}$. The vector and scalar spectral functions, $\rho_{\mathrm{v}}, \rho_{\mathrm{s}}$, sum up to zero and hence are necessarily negative for some momentum regime,

$$
\int_{-\infty}^{\infty} d \omega \rho_{\mathrm{v}}(\omega,|\boldsymbol{p}|)=0, \quad \int_{-\infty}^{\infty} d \omega \rho_{\mathrm{s}}(\omega,|\boldsymbol{p}|)=0 .
$$

Using specific projection operators for the chirally symmetric and/or static $(|\boldsymbol{p}|=0)$ case, one can define positive semidefinite combinations of $\rho_{4}, \rho_{v}$ and $\rho_{s}$ using Eq. (9); see e.g., Ref. [6] for details. In this first study, here, we restrict ourselves to the reconstruction of the component $\rho_{4}$ only. The corresponding left-hand side of Eq. (7) is then also given by the $\gamma_{4}$ component of the quark propagator, i.e.,

$$
\begin{aligned}
& S_{4}\left(i \omega_{p},|\boldsymbol{p}|\right) \\
& \quad=\frac{i \omega_{p} C\left(\omega_{p},|\boldsymbol{p}|\right)}{\omega_{p}^{2} C\left(\omega_{p},|\boldsymbol{p}|\right)^{2}+\boldsymbol{p}^{2} A\left(\omega_{p},|\boldsymbol{p}|\right)^{2}+B\left(\omega_{p},|\boldsymbol{p}|\right)^{2}} .
\end{aligned}
$$

This component has the advantage of being antisymmetric in $\omega_{p}$ and the corresponding part of the spectral function being symmetric in $\omega$, which means that we may restrict ourselves in Eq. (7) to positive frequencies and arrive, using Eq. (8), at a purely imaginary kernel,

$$
S_{4}\left(i \omega_{p}, \boldsymbol{p}\right)=i \int_{0}^{\infty} d \omega^{\prime} \frac{2 \omega_{p} \rho_{4}\left(\omega^{\prime}, \boldsymbol{p}\right)}{\omega_{p}^{2}+\omega^{\prime 2}},
$$

which is easily implemented numerically for the spectral reconstruction; i.e., we will directly formulate the inverse problem in imaginary frequency space, since the rational kernel in Eq. (12) suppresses spectral information much less than its Euclidean counterpart and thus is ideally suited for use in spectral reconstructions.

\section{BAYESIAN SPECTRAL RECONSTRUCTION}

\section{A. Bayesian inference}

In this study, we use the concept of Bayesian inference to invert the relation of Eq. (12) numerically. The quark two-point function $S_{4}$ is evaluated along discretized imaginary frequencies $\omega_{p}$ with $p \in\left[1 \ldots N_{\text {data }}\right]$, while the spectral function is resolved along $N_{\omega}$ bins in realtime frequencies $\omega_{l}^{\prime}$,

$$
-i S_{4}\left(i \omega_{p}\right)=\sum_{l=1}^{N_{\omega}} \frac{2 \omega_{p}}{\omega_{p}^{2}+\omega_{l}^{\prime 2}} \rho_{l} .
$$

The data here are obtained from a functional QCD computation. Thus they can be evaluated at an arbitrary number of points $N_{\text {data }}$ but contain a finite numerical error $\Delta S_{4}$ due to the evaluation of intermediate integrals in e.g., Eq. (3). Therefore, for any finite $N_{\text {data }}$ and $\Delta S_{4}$, a naive $\chi^{2}$ fit of the $N_{\omega}$ parameters $\rho_{l}$ would lead to an infinite number of degenerate solutions, all reproducing the data within their uncertainty. Bayesian inference in the form of Bayes theorem,

$$
P\left[\rho \mid S_{4}, I\right]=\frac{P\left[S_{4} \mid \rho, I\right] P[\rho \mid I]}{P\left[S_{4} \mid I\right]},
$$

provides a systematic prescription of how to regularize the otherwise underdetermined $\chi^{2}$ fit. It does so by incorporating additional prior information $I$ on the spectrum, in addition to the correlator data $S_{4}$. The posterior $P\left[\rho \mid S_{4}, I\right]$ for a test function $\rho$ denotes its probability to be the correct spectrum, given the computed data and prior information. It is proportional to the likelihood probability $P\left[S_{4} \mid \rho, I\right]$ and the prior probability $P[\rho \mid I]$.

The former encodes how the correlator has been obtained and in case of stochastically independent sampled data is related to the standard $\chi^{2}$ fitting functional

$$
P\left[S_{4} \mid \rho, I\right]=\exp [-L]
$$

with likelihood 


$$
\begin{aligned}
L= & \frac{1}{2} \sum_{p, q=1}^{N_{\text {data }}}\left(S_{4}\left(i \omega_{p}\right)-S_{4}^{\rho}\left(i \omega_{p}\right)\right) \\
& \times C_{p q}^{-1}\left(S_{4}\left(i \omega_{q}\right)-S_{4}^{\rho}\left(i \omega_{q}\right)\right) .
\end{aligned}
$$

Here, $S_{4}^{\rho}\left(i \omega_{p}\right)$ denotes the correlator according to the current choice of test function via Eq. (13), and $C_{p q}$ is the covariance matrix of the actual correlator data $S_{4}$. Note that we have formulated the inverse problem in the imaginary frequency domain and not based on Euclidean data. The reason is that the exponentially damped integral kernel and the information loss associated with it lead to much worse reconstruction results than the rational Källen-Lehmann kernel used here (for an explicit demonstration, see Refs. [39,51]). Note also that in the case of the Euclidean kernel it was found that in order to compute the propagator from a test spectral function $\rho$ with high accuracy one needs to deploy a logarithmic frequency grid [52].

Since furthermore in our case the correlator is computed and not sampled, we will assign an estimated diagonal covariance matrix to the discrete $S_{4}$ with constant relative errors $\Delta S_{4} / S_{4}=$ const. $L[\rho]$ possesses many flat directions, which translate into the nonuniqueness of the maximum likelihood $\chi^{2}$ approach.

The second and decisive term in the numerator of Eq. (14) is the prior probability, which acts as a regulator to the likelihood probability, lifting the flat directions of $L$. It tells us how compatible the test function $\rho$ is with available prior information. Conventionally, it is expressed as

$$
P[\rho \mid I]=\exp [\alpha S[\rho, m]]
$$

where $\alpha$ represents a so-called hyperparameter, which weighs the influence of the data versus the prior. Prior information is encoded in $P[\rho \mid I]$ in two ways, on the one hand via the functional form of $\mathrm{S}$ itself and via the so-called default model $m(\omega)$, which by definition is the most probable spectrum in the absence of data; i.e., it represents the unique extremum of $S[\rho, m]$.

Different implementations of the Bayesian strategy differ not only in the regulator $S$, which they employ, but also in how the hyperparameter $\alpha$ is treated, as well as how the most probable spectrum

$$
\left.\frac{\delta P\left[\rho \mid S_{4}, I\right]}{\delta \rho_{l}}\right|_{\rho=\rho^{\text {Bayes }}}=0
$$

is determined numerically. Note that different Bayesian prescriptions can yield different results, as long as $N_{\text {data }}$ and $\Delta S_{4}$ are finite. Only in the "Bayesian continuum limit," all methods, if implemented correctly, must converge to the same result. It is therefore important to test how reconstructions change toward this limit using e.g., mock data tests.
In this study, we will work with quarks at high temperature, where the spectral functions are assumed to be positive definite, a property which in turn will enter as prior information.

The popular Maximum Entropy Method for positive definite spectra proposes using the Shannon-Jaynes entropy as regulator, a choice justified by arguments from two-dimensional image reconstruction,

$$
S_{\mathrm{SJ}}=\int d \omega\left(\rho-m-\rho \log \left[\frac{\rho}{m}\right]\right) .
$$

One carries out the reconstruction multiple times with different values of $\alpha$ and then averages these intermediate results $\rho_{\alpha}$ self-consistently over a probability distribution for $\alpha, P\left[\alpha \mid S_{4}, I\right]$. In the standard implementation by Bryan, the functional space, from which the test function $\rho$ is chosen, is manually limited to a dimensionality of $N_{\text {data. }}$. It has been shown that the extremum of $P\left[\rho \mid S_{4}, I\right]$ in general is not contained in this choice of search space [53], which may result in slow convergence. In addition, the determination of $P\left[\alpha \mid S_{4}, I\right]$ relies on a Gaussian approximation (see also the discussion in Appendix C).

In this study, we instead use a more recent Bayesian approach to spectral function reconstruction that has been developed with the one-dimensional inverse problem of Eq. (13) in mind. Its regulator functional reads

$$
S_{\mathrm{BR}}=\int d \omega\left(1-\frac{\rho(\omega)}{m(\omega)}+\log \left[\frac{\rho(\omega)}{m(\omega)}\right]\right) .
$$

The hyperparameter $\alpha$ is treated in a Bayesian fashion, in that we assume full ignorance of its values $P[\alpha]=1$ and integrate it out a priori,

$$
P\left[\rho \mid S_{4}, I(m)\right]=P\left[S_{4} \mid I\right] \int_{0}^{\infty} d \alpha P[\rho \mid I(\alpha, m)] .
$$

In addition, we also require that $L=N_{\text {data }}$ as the correct spectrum, on average, would lead to such a value. The most probable spectrum is then obtained from carrying out a numerical optimization on $P\left[\rho \mid S_{4}, I(m)\right]$ according to Eq. (18). In contrast to the MEM, we do not restrict the search space and use a pseudo-Newton method (limited Broyden-Fletcher-Goldfarb-Shanno) to find the global extremum in the full $N_{\omega}$ dimensional search space.

The regulator $S_{\mathrm{BR}}$ was derived with the goal being to keep its influence on the outcome of the reconstruction to a minimum; i.e., it shall influence the reconstruction as weakly as possible and "let the data speak." Comparing $S_{\mathrm{BR}}$ to $S_{\mathrm{SJ}}$ or the quadratic prior, one finds that far away from the extremum $\rho=m, S_{\mathrm{BR}}$ shows the weakest curvature. While this makes it easy for structures encoded in the data to manifest themselves in the reconstruction, it also means that common artifacts associated with inverse problems, such as Gibbs ringing, are only weakly suppressed.

In turn, if the structures of physical interest are spectral peaks, as will be the case in the following, we have to make 


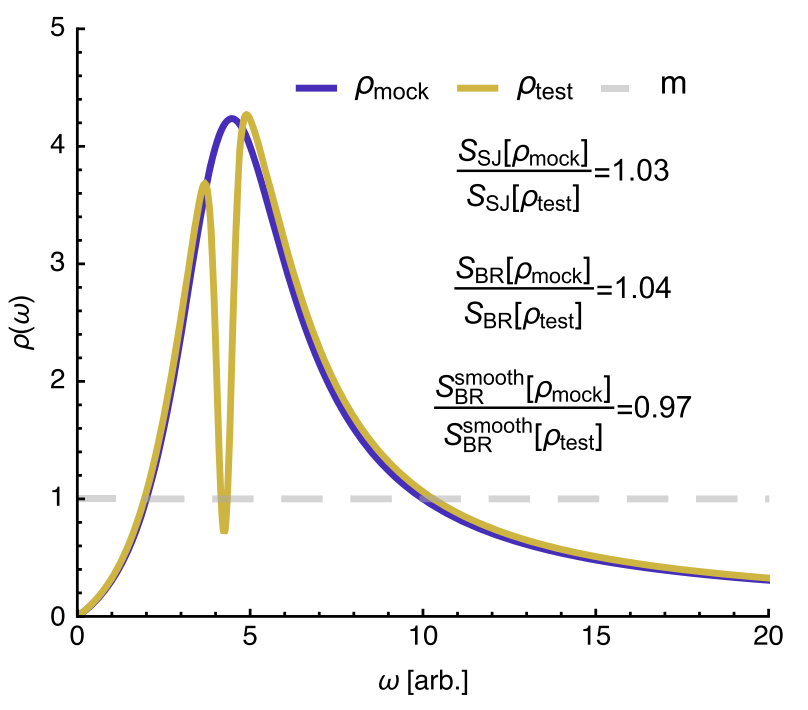

FIG. 3. Illustration of the susceptibility of the regulators $S_{\mathrm{BR}}$ and $S_{\mathrm{SJ}}$ on ringing artifacts. If a mock spectrum $\rho_{\text {mock }}$ (dark blue) contains a broad feature, then introducing a small wiggle leads actually to a smaller penalty.

sure that we unambiguously distinguish ringing from genuine features. Due to its restricted search space, the MEM usually produces smooth features, and it is considered safe from ringing. This impression is unfortunately incorrect on the level of the regulator as illustrated in Fig. 3. For a flat default model $m=1$, we compare the penalty assigned to a function $\rho_{\text {mock }}$ with a single broad feature, as well as a similar function with an additional wiggle close to its peak $\rho_{\text {test }}$. The area under both spectra has been kept the same. What one finds is that $S_{\mathrm{BR}}$ and $S_{\mathrm{SJ}}$ assign a lower penalty to the distorted curve, even though the former was explicitly derived with a smoothness axiom. What distinguishes the two curves is of course their arc length, which increases for every additional wiggly structure.

Hence, we introduce a new BR-type regulator, which penalizes arc length $\ell=\int d \omega \sqrt{1+(d \rho / d \omega)^{2}}$ explicitly. In order to leave as much of the original form of $S_{\mathrm{BR}}$ intact, we add a $\ell^{2}$ term for both the spectrum and default model and subtract unity. ${ }^{1}$ The result is

$$
\begin{aligned}
S_{\mathrm{BR}}^{\text {smooth }}= & \int d \omega\left(-\gamma\left(\frac{d \rho(\omega)}{d \omega}-\frac{d m(\omega)}{d \omega}\right)^{2}\right. \\
& \left.+1-\frac{\rho(\omega)}{m(\omega)}+\log \left[\frac{\rho(\omega)}{m(\omega)}\right]\right) .
\end{aligned}
$$

\footnotetext{
${ }^{1}$ This naive extension of the standard BR method actually violates the axiom of scale invariance, which, however, may be recovered by introducing a further default model related function $h(\omega)$, which encodes the uncertainty in the derivative of the default model, similar to the case of the generalized BR method [39].
}

The new hyperparameter $\gamma$ reflects the freedom we have to adjust the influence of the penalty term in addition to setting the overall strength of the prior information, weighted against data via $\alpha$. In this study, we will use the following strategy to set $\gamma$ : As the intent of introducing the additional penalty term is to remove ringing artifacts, and we reduce to the standard BR method in the case $\gamma=0$, we will carry out mock data analyses with a realistic mock spectrum (see Sec. III B) and select the lowest value of $\gamma$ for which artificial ringing is completely suppressed. This strategy of using mock training data to select a hyperparameter is similar to those used in inference via supervised machine learning. From our mock data tests, we find $\gamma=\frac{1}{2}$ to be appropriate.

In the presence of the derivative term, we are not any more able to analytically compute the $\alpha$ dependent normalization of the prior probability and thus cannot marginalize $\alpha$, as in done in Eq. (21). Thus, we revert to handling $\alpha$ in the same way as in the "historic MEM" prescription, in which one adjusts $\alpha$ in order that $\left(L-N_{\text {data }}\right)<10^{-1}$.

As we will see also explicitly in the mock data test in the following section, this new regulator with $\gamma=\frac{1}{2}$ succeeds in efficiently suppressing ringing artifacts. At the same time, peaks encoded in the correlator become more washed out, and it requires more data points and smaller errors to resolve these peaks to the same accuracy as with the original BR method. Hence, our strategy is the following. Using the smoothening prior, we will identify in which part of the spectrum actual peak structures reside and then extract their features using the standard BR approach.

The reliability of the reconstruction can be estimated in three different ways. Since in Bayes's theorem in Eq. (14) data and prior information enter, we need to understand the dependence of the reconstruction result on that input. For the former, we can vary the number of provided data points and carry out a bootstrap jackknife resampling analysis of the correlator. Since here we do not use sampled data, we will instead successively lower the assigned error on the data and observe convergence toward the Bayesian continuum limit. The dependence on the prior can be estimated by repeating the reconstructions with different choices of the default model, for which we choose a flat function $m(\omega)=m_{0}$ and vary $m_{0}=\{0.1,0.5,1.0,5.0,10\}$.

Bayesian methods also provide another measure for the robustness of the reconstruction

$$
\left\langle\delta \rho^{2}\right\rangle_{I} \approx-\int_{I} d \omega d \omega^{\prime}\left(\delta^{2} Q / \delta \rho^{2}\right)^{-1} / \int_{I} d \omega d \omega^{\prime},
$$

based on the curvature of the optimization functional $Q\left[\rho, S_{4}, I\right]=\log \left[P\left[\rho \mid S_{4}, I\right]\right]$. In previous works, it has been found that the actual dependence on the variation of data and prior information is often larger than indicated by this quantity. One possible reason for this discrepancy is 
the need for two assumptions in the derivation of Eq. (23), namely that the posterior is both highly peaked and may be approximated by a Gaussian. In the following, we will show error bands for the BR method that are obtained from the variation of the default model and the curvature measure, where the former dominates.

\section{B. Mock data tests}

Previous studies [13] deploying a MEM-type approach hinted at the presence of two-peak structures at positive frequencies in the quark spectral function in the Landau gauge at finite temperature. One of the peaks resides at the origin, one at a distance from the origin significantly larger than its width. On the other hand, it is was not excluded that further peaked features may be present. Therefore, we must ascertain how well our reconstruction method will be able to resolve different structures given a certain quality of input data, for which we turn to a mock data analysis.

In the following, we compute the Euclidean frequency correlator $S_{4}$ according to Eq. (7) based on mock spectra with different peak contents. This continuous function is then evaluated at $N_{\text {data }}^{\text {mock }}=128$ discretely spaced imaginary frequencies between $\omega_{p} \in[0,8] \mathrm{GeV}$. These ideal data are fed to the reconstruction algorithm undistorted but are assigned a constant relative error $\Delta D / D$. Increasing the number of data points beyond 128 has not shown significant improvements down to $\Delta D / D=10^{-8}$.

By successively lowering $\Delta D / D$, we have investigated the approach to the Bayesian continuum limit for fixed $N_{\text {data }}$ as explicitly shown in Appendix A. In the following, we will showcase only two of these reconstructions for each mock spectrum, which are relevant for the discussion. One is the best possible outcome using a particular Bayesian implementation, i.e., using a very small $\Delta D / D=10^{-8}$. The other is the realistic case $\Delta D / D=10^{-3}$, which corresponds to the precision easily obtainable in realistic Dyson-Schwinger computations [52].

As a first step, let us have a look at a two-peak scenario with one structure located at the origin and a second at $\omega=2 \mathrm{GeV}$, as shown by the grey curve in Fig. 4. The two solid curves of different brightness show the reconstructions based on either the smoothed BR method (top) or its original implementation (bottom). The darker curve corresponds to the error $\Delta D / D=10^{-3}$, and the lightest color corresponds to $\Delta D / D=10^{-8}$. The light colored bands around the curves refer to the corresponding dependence on the choice of the prior.

We find that already at $\Delta D / D=10^{-3}$ the shape of the peak at the origin is very well reproduced. The smooth BR method shows only minute deviations from the actual functional form up to $\omega=0.2 \mathrm{GeV}$, while the standard BR method still exhibits some ringing artifacts. In addition, the position of the second peak is reproduced accurately within $15 \%-20 \%$ for both variants of the BR method. The
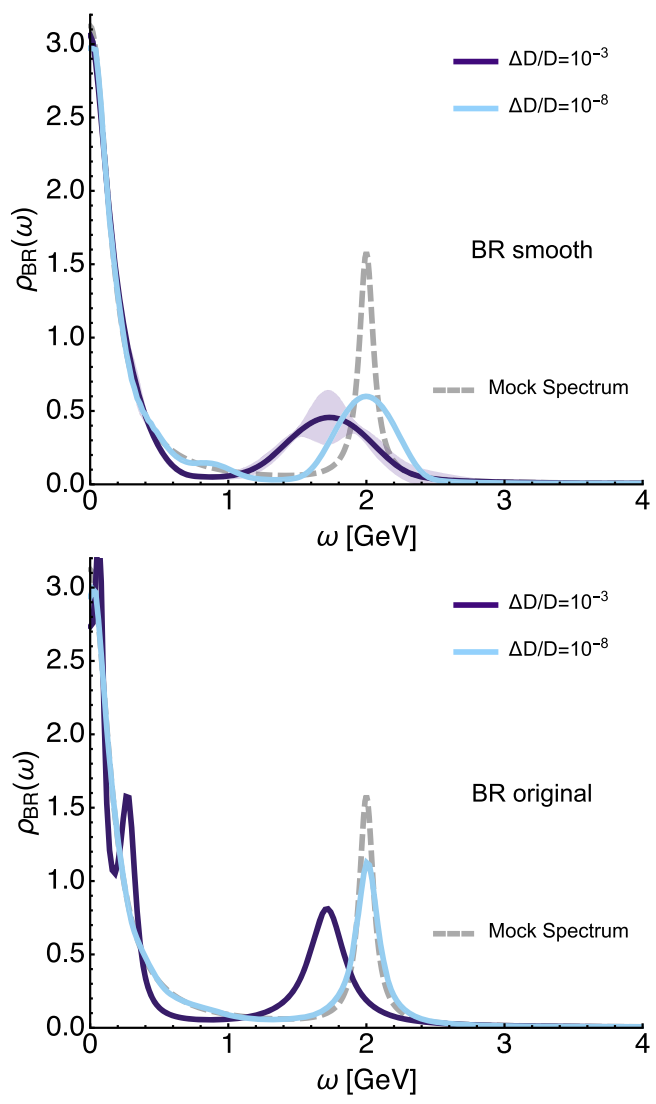

FIG. 4. Mock data analysis of reconstruction reliability for a two-peak scenario (grey dashed). Both the results for the smooth $\mathrm{BR}$ (top) as well as the original BR (bottom) method are shown. The dark curve denotes realistic errors $\Delta D / D=10^{-3}$, while the light curve denotes the close to optimal result for $\Delta D / D=10^{-8}$. Already at $\Delta D / D=10^{-3}$, the lowest peak is very well reproduced by the smooth BR method, while the standard BR shows some ringing artifacts. The position of the second peak is estimated within $15 \%-20 \%$ of the correct value. At $\Delta D / D=10^{-8}$, both methods are devoid of ringing artifacts, and the position of the second peak is faithfully reproduced.

width, as expected, is overestimated in both cases, and the effect is stronger by construction for the smooth BR method.

It is evident that lowering the errors consistently improves the reconstruction of both the position and width of the second peak. At $\Delta D / D=10^{-8}$, both methods reproduce faithfully the position also of the second peak; at the same time, also the estimation of the width improves significantly. With such high precision data, none of the two methods shows any ringing artifacts.

We continue with a more challenging scenario, where one of the peaks is split into two structures. In particular, here, we consider the emergence of an additional structure close to the origin. If the peak at large frequencies is well separated from these, then again neither the conventional nor the smooth BR method is challenged in identifying the three structures as seen by the results of Fig. 5. Of course, 

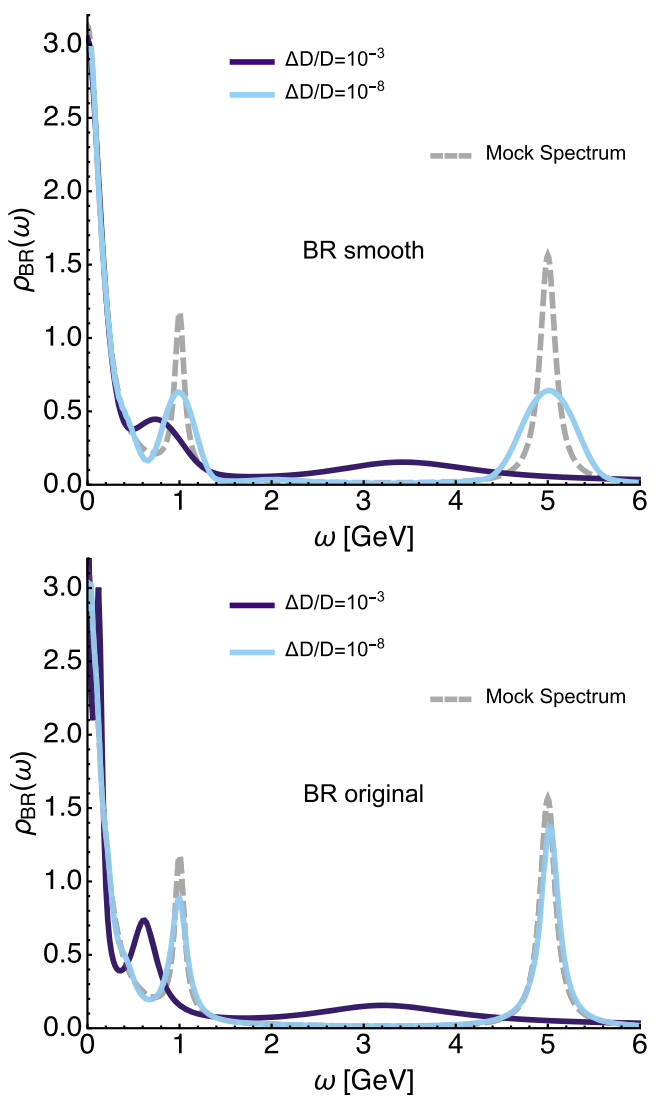

FIG. 5. Mock data analysis of reconstruction reliability for a three-peak scenario (grey dashed) with two peaks closely positioned off the origin. Both the results for the smooth BR (top) as well as the original BR (bottom) method are shown.The dark curve denotes realistic errors $\Delta D / D=10^{-3}$, while the light curve denotes the close to optimal result for $\Delta D / D=10^{-8}$.

even though at $\Delta D / D=10^{-3}$ its presence is detected, a quantitative determination of the features of the third peak requires much smaller data uncertainty.

An even more difficult scenario for any Bayesian reconstruction arises in the presence of an additional peak positioned closely to the one off the origin. Here, close is understood as a distance which is comparable to the width of the peaks, as shown in Fig. 6 (grey dashed). Both methods struggle to identify the split between the two peaks even at optimal conditions $\Delta D / D=10^{-7}$. On the other hand, it is important to note that the reconstructions of the two different methods show qualitatively different behavior in contrast to all previous scenarios. While before the shapes of the peaks in both the conventional and smooth $\mathrm{BR}$ methods agreed, here we see that at $\Delta D / D=10^{-7}$ the original BR method starts to show a distorted peak.

Let us summarize the findings of the preceding section. Our goal in introducing a smooth BR method was to enable the identification of ringing artifacts in the standard BR method. We find in the mock analysis that this goal is achieved, as the former exhibits no sign of artificial wiggles. At the same time, both methods are able to
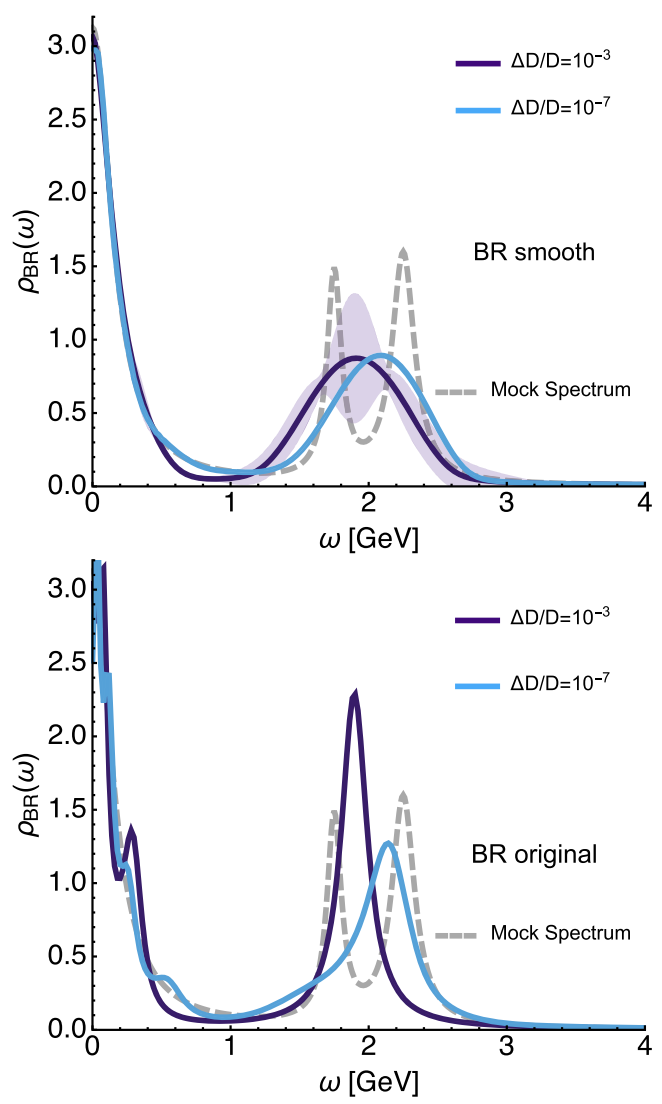

FIG. 6. Mock data analysis of reconstruction reliability for a three-peak scenario (grey dashed) with two peaks closely positioned off the origin. Both the result for the smooth BR (top) as well as the original BR (bottom) method are shown. The dark curve denotes realistic errors $\Delta D / D=10^{-3}$, while the light curve denotes the close to optimal result for $\Delta D / D=10^{-7}$.

identify the presence of peaks, as long as they are separated by more than twice their width. For more closely situated structures, even going to unrealistic conditions, such as $\Delta D / D=10^{-7}$, does not provide a satisfactory resolution. In practice, as detailed in the next section, we find neither signs of numerical ringing artifacts nor any indications of the presence of a third peak in our spectra reconstructed with the standard BR method.

Quantitative estimates of spectral features will in the following always be carried out by the standard BR method, after having made sure via the smooth BR method that the peak structure in question is not a ringing artifact. For realistic data i.e., $\Delta D / D \sim 10^{-3}$, we find that the BR method will allow us to extract the peak position with up to around $15 \%-20 \%$ uncertainty, while peak heights are more difficult to estimate with 30\%-50\% uncertainty. Additional comparison with data manually made more sparse will tell us how much of the observed temperature dependence of spectral features is attributable to the medium and how much again has to be understood as a methods artifact. 


\section{RESULTS}

In this section, we apply the previously tested Bayesian approaches to actual correlator data $S_{4}$ obtained from Dyson-Schwinger computations. We first reanalyze the rainbow-ladder model approach described at the end of Sec. II A. In this simple truncation, the determination of the correlator is computationally cheap, and numerical errors are easily reduced to $\Delta D / D<10^{-3}$. Subsequently, we discuss our main results based on the truncation scheme with $N_{f}=2+1$ unquenched light flavors, back coupled to the Yang-Mills sector.

\section{A. Rainbow-ladder truncation}

\section{Chiral limit}

Let us first discuss the case of the chiral limit, which generates a second order phase transition at a critical temperature of $T_{c}=0.142 \mathrm{GeV}$. We have evaluated the corresponding $S_{4}$ along Matsubara frequencies at 12 temperatures above $T_{c}$ in the range of $T \in[0.15,0.60] \mathrm{GeV}$. Due to the used cutoff $\Lambda=100 \mathrm{GeV}$ and the discrete nature of the Matsubara frequencies, this corresponds to $N_{\text {data }} \in$ $[106,27]$. The correlator computations have been checked to carry a numerical error of less than $\Delta D / D \leq 10^{-3}$, so we can assign a corresponding diagonal correlation matrix to it. The imaginary frequency dependence of $S_{4}$ at vanishing spatial momentum is plotted in Fig. 7.

We deploy the conventional and smooth BR method with a frequency discretization of $N_{\omega}=1000$ in the interval $\omega \in$ $[0,20]$ for reconstructing the quark spectral function. The default model is set to a constant $m(\omega)=m_{0}$, and we carry out the reconstruction with the different choices $m_{0}=$ $\{0.1,0.5,1.0,5.0,10\}$. The variance in the outcome is

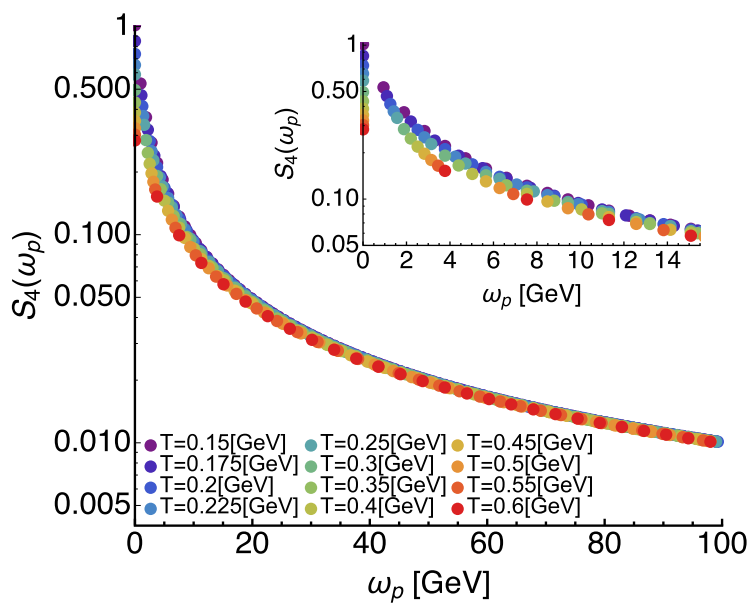

FIG. 7. Zero momentum imaginary frequency quark correlator in the chiral limit at finite temperature from the rainbow-ladder truncation. Above $\omega_{p}>30 \mathrm{GeV}$, all correlators already lie very close to the $T=0$ curve. Numerical errors are smaller than the data-point markers. taken as the basis for the error bands depicted in the subsequently shown plots.

Our intention in using two different Bayesian methods is to cross-check that the peak structures observed with the standard BR method are indeed physical and not related to numerical ringing. In the following, we will therefore first discuss representative reconstructions for both of the algorithms and, if no signs of ringing are encountered, remain with the standard $\mathrm{BR}$ method, as it allows us to more accurately extract the properties, such as the position and weight of individual spectral features.

In Fig. 8, we present the zero momentum reconstruction of the quark spectral function for different temperatures. Note that since we explicitly use the symmetry of the spectral function in Eq. (12) the position of the peak at positive frequency $\omega_{+}$is mirrored exactly in the negative frequency domain. In the following, it is therefore sufficient to discuss the positive frequency regime only.

In the top panel, the outcome of the smooth BR method is shown, while the bottom panel corresponds to the conventional one. Note that there are no signs of numerical ringing present and the number of peaks agrees between the
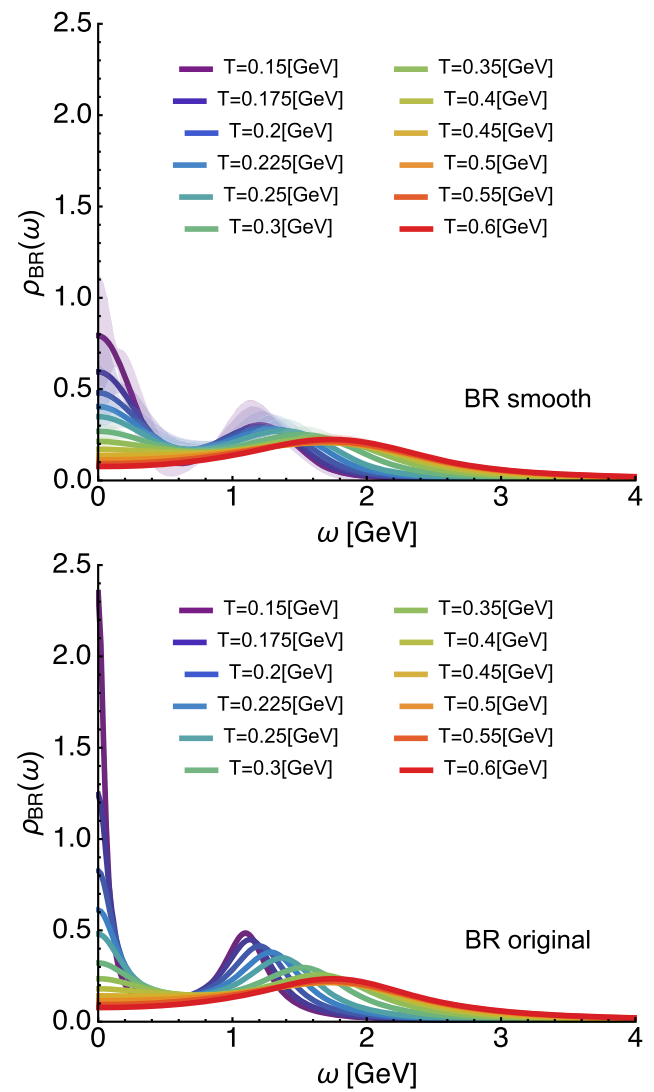

FIG. 8. Zero momentum reconstruction of the quark spectral function in the chiral limit at finite temperature using the smooth BR (top) and the original BR (bottom) method. Both methods show a clear indication for the presence of two-peak structures at low temperature. The structure at the origin weakens with increasing $T$, as does the higher lying peak, while also shifting. 


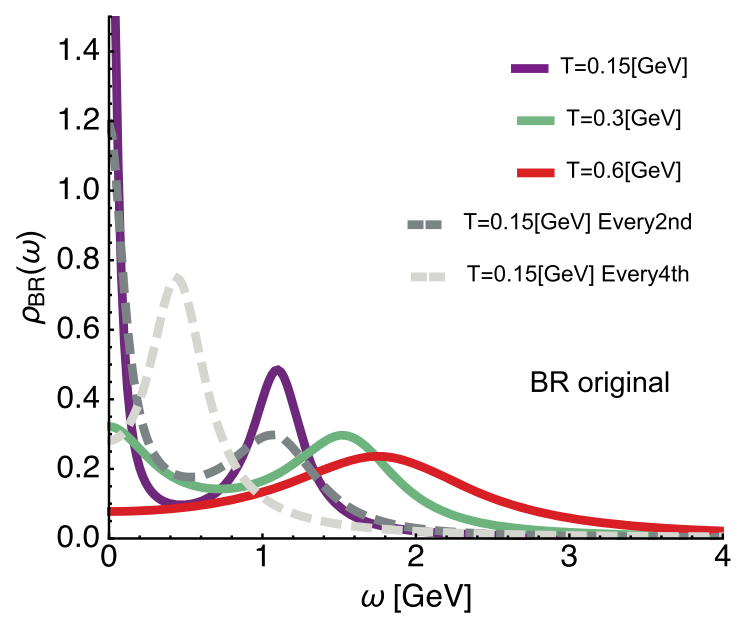

FIG. 9. Test of the reliability of the standard BR reconstruction at different temperatures based on the correlator manually made more sparse at $T=0.15 \mathrm{GeV}$. Using only every second data point of the original $N_{\text {data }}=106$ leads to diminished peak heights (dark grey) but does not influence peak positions. When made more sparse to every fourth, the Bayesian method does not recover the peaks satisfactorily anymore (light grey). Nonetheless, clear in-medium modification at $T>0.15 \mathrm{GeV}$ is observed.

two methods. We learn that at low temperatures at least up to $T \lesssim 0.25 \mathrm{GeV}$ two well-defined peak structures exist. One is located at the origin, and one above $1 \mathrm{GeV}$. With increasing temperature, the height of the low lying peak decreases continuously and seems to asymptote to a finite value. The higher lying peak shows a clear tendency to move to higher frequencies, while broadening at the same time.

The reconstructions at different temperatures are based on a data set with different $N_{\text {data }}$. Thus, before we continue to a more quantitative inspection of the spectra, we need to make sure that, using the standard BR method, we can disentangle the actual in-medium modification from the effects of a reduction of data points. To this end, we perform the following test: We take the lowest temperature data set with $N_{\text {data }}=106$ in imaginary frequencies and make it more sparse by hand. Due to the discrete nature of the Matsubara frequencies, this corresponds to a situation where the $T=0.15 \mathrm{GeV}$ spectrum would be encoded in a correlator evaluated at $T=0.3 \mathrm{GeV}$ or $T=0.6 \mathrm{GeV}$ respectively. In Euclidean time, this corresponds to constructing the appropriate reconstructed correlator [54]. From similar tests performed in previous Bayesian studies, we expect that with the decreasing number of data points the resolution of peaks diminishes, eventually inducing changes in the position and width of the reconstructed features.

And indeed, as shown in Fig. 9, we find that going from $N_{\text {data }}=106$ to $N_{\text {data }}=53$ (dark grey dashed) leads to a visible weakening of the peak structures, while their position remains unaffected. Comparing to the reconstruction from actual $T=0.3 \mathrm{GeV}$ with the same lower number of data
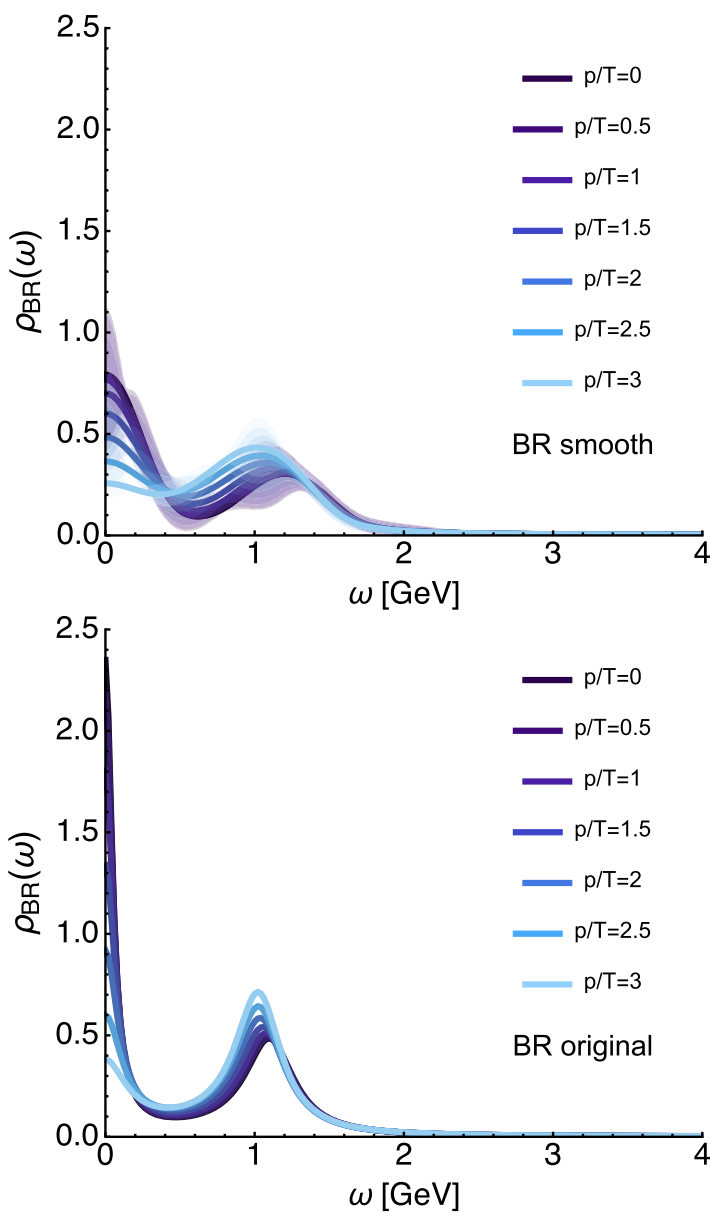

FIG. 10. Low temperature $T=0.15 \mathrm{GeV}$ reconstructions of the quark spectral function at different momenta using the smooth $\mathrm{BR}$ (top) and the original BR (bottom) method. We find clear indications of a two-peak structure. Both peak heights decrease for larger momenta; the one close to the origin decreases, however, much more rapidly. A shift of the second peak to higher frequencies is observed.

points (green solid), however, shows clear differences. Both the diminishing of the lowest lying peak height as well as the shifting of the second peak to higher frequencies can thus be attributed to genuine in-medium effects. Interestingly, the direction of change in the peak position is opposite to that sketched in Refs. [13,14]. At $T=0.6 \mathrm{GeV}$, i.e., $N_{\text {data }}=26$, the data made more sparse do not allow the reconstruction of the two peaks at all, and we must assume that the reconstruction is not trustworthy at this point.

The investigation of the effects of finite momentum on the quarks does not suffer from a similar ambiguity, since for fixed $T$ neither the number of data points nor the relative errors change. In Fig. 10, we show reconstructed spectra at $T=150 \mathrm{MeV}$ over a range of momenta $|\boldsymbol{p}| / T=\left\{0, \frac{1}{2}, 1, \frac{3}{2}, 2, \frac{5}{2}, 3\right\}$.

As seen before at low temperature, both reconstruction approaches unambiguously show the presence of two peaks. One is located around the origin, and another one 

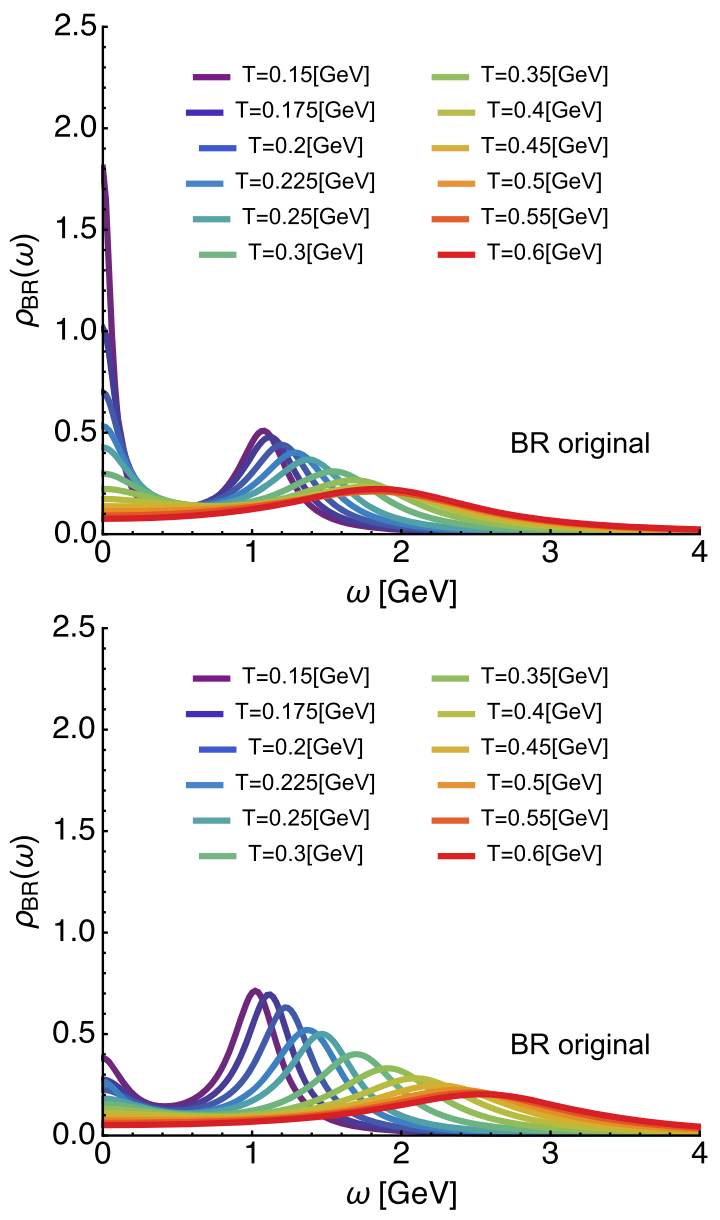

FIG. 11. Intermediate momentum $|\boldsymbol{p}| / T=1$ (top) and $|\boldsymbol{p}| / T=$ 3 (bottom) reconstructions of the quark spectral function at different temperatures using the original BR method. The characteristic decrease in the peak height around the origin as well as the shift and broadening of the higher lying peak structure is clearly visible.

is positioned close to $\omega \approx 1 \mathrm{GeV}$. Increasing the momentum to $|\boldsymbol{p}| / T=3$ induces changes in the spectrum that are of the same qualitative nature as those from increasing temperature. The peak at the origin decreases significantly in area, while not extending further toward higher $\omega$. The second peak diminishes much more weakly and is seen to shift to higher frequencies, as expected from the naive momentum dependence of the dispersion relation.

For visualization purposes, we present in Fig. 11 the reconstructions at a fixed intermediate momentum $|\boldsymbol{p}| / T=$ 1 (top) and $|\boldsymbol{p}| / T=3$ (bottom) for all different temperatures investigated. All the effects on the peak around the origin as well as the second peak at finite frequencies as discussed above are clearly visible here.

We continue with a quantitative analysis of the inmedium modification of the quark spectral features. Two quantities of interest here are the position of the higher lying peak, denoted with $\omega_{+}$, and the height of the peak around the origin, referred to as $Z_{0}$. Both are shown in
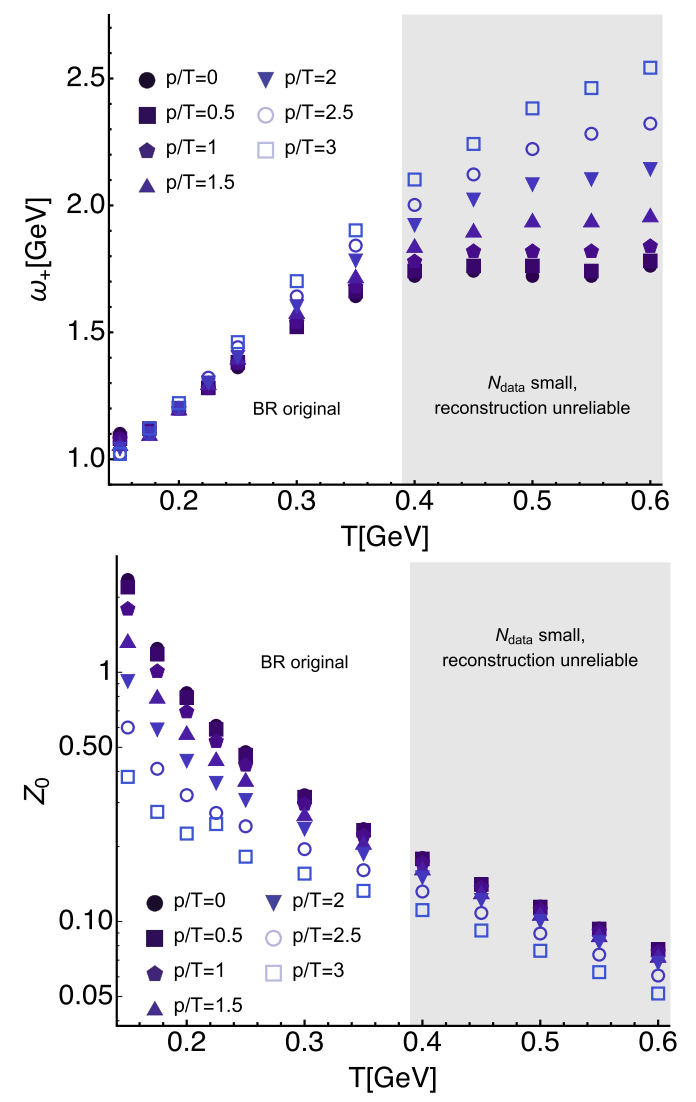

FIG. 12. (Top) position of the higher lying peak for different temperatures and momenta. At intermediate temperatures, where the reconstruction is reliable, we find a linear rise, which flattens off, once the number of data points becomes too small. (Bottom) Amplitude of the lowest lying peak, which we find to decrease monotonously.

Fig. 12. For $\omega_{+}$, the expectation from resummed hardthermal loop perturbation theory at small $|\boldsymbol{p}| / T \ll 1$ is a linear dependence on the temperature $\omega_{ \pm}^{\mathrm{HTL}}=m_{T} \pm \frac{|p|}{3}$ with thermal quark mass $m_{T} \propto T$. While at low temperatures and small momenta we see a rise stronger than linear, at intermediate $T$, our $\omega_{+}$indeed shows a behavior compatible with a linear increase. Consistent with our conclusions from the test based on input data that was manually made more sparse, for temperatures much higher than $T=0.3 \mathrm{GeV}$, the reconstruction becomes unreliable, and at the same time, we see that the linear rise abates and goes over to a constant.

The behavior found here is again different from that presented in Ref. [14]. First, we find that the peak at nonzero frequency $\omega_{+}$monotonously moves to larger frequencies with increasing temperature in contrast with the previously reported behavior, where the function $\omega_{+}(T)$ shows a minimum shortly above $T_{c}$. The second difference is related to the height of the zero frequency peak, which in the temperature range investigated does not go to zero but stays finite. Since the high temperature regime is not reliably captured due to the sparseness of the Matsubara 


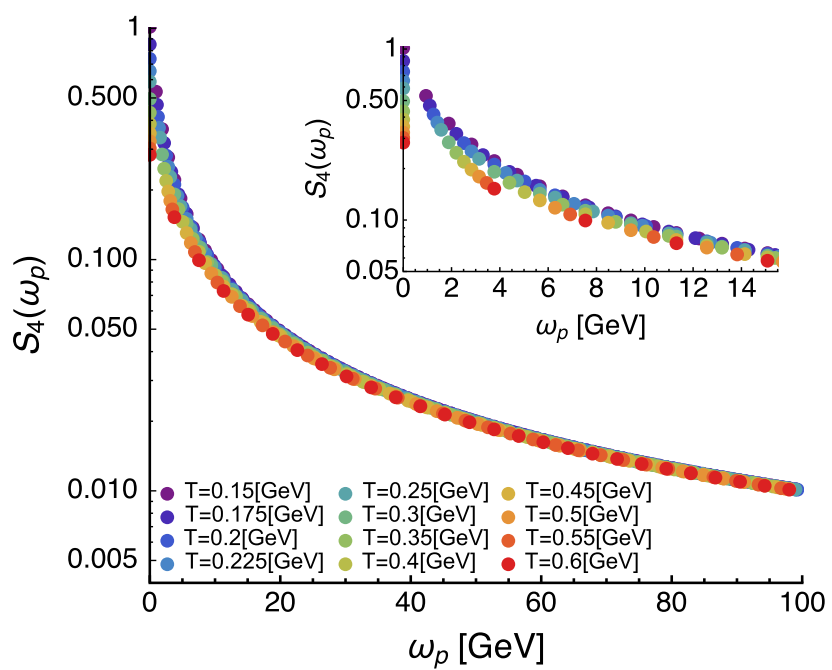

FIG. 13. Zero momentum imaginary frequency quark correlator at finite mass and finite temperature from the rainbow-ladder truncation. Above $\omega_{p}>30 \mathrm{GeV}$, all correlators already lie very close to the $T=0$ curve. Numerical errors are smaller than the data-point markers.

frequencies, we cannot yet conclude whether it eventually vanishes after all.

In all quantitative statements, we need to keep in mind that our current numerical precision for the correlator data is limited to $\Delta D / D=10^{-3}$. While we are confident, judging from the mock data tests, that the number of peaks and the direction of changes with temperature are correctly captured, it is fathomable that the peak position may still change by up to $20 \%$ if the errors are further reduced. The width of the peaks carries at least the same uncertainty at this point along the Bayesian continuum limit.

Another word of caution is in order regarding the quantitative determination of the peak height. While peak positions are among the most robust features to reconstruct, the peak height stills shows a significant variation comparing e.g., the standard and smooth BR outcomes in Fig 14. From our mock data analysis, we understand that the smooth BR method consistently underestimates the peak height, while the standard method works rather well at determining this spectral property even in a realistic scenario with $\Delta D / D=$ $10^{-3}$ (at least for the lowest lying structure at $\omega=0$ ). We further see in Fig. 9 that, while the change in available data points in Fig. 9 leads to a significant change in the peak height, the majority of the observed effect still may be attributed to thermal effects. The uncertainties could be significantly reduced by instead considering the area under the spectral peak, which we found shows more consistency between the different Bayesian methods than the peak height alone.

\section{Finite mass case}

We proceed with a second set of correlators $S_{4}$, for which the current quark mass has been set to $m_{q}=3.7 \mathrm{MeV}$ at a
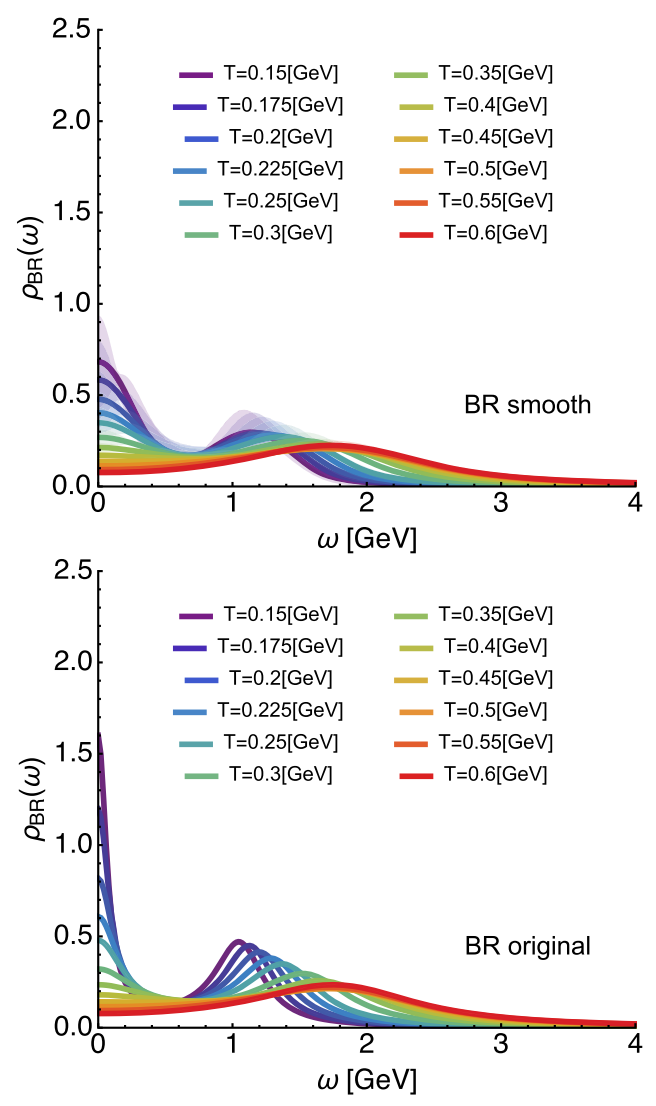

FIG. 14. Zero momentum reconstruction of the quark spectral function at finite mass and finite temperature using the smooth BR (top) and the original BR (bottom) methods. We find qualitatively the same behavior as in the chiral case, the main difference being the height of the lowest lying peak at low temperature.

renormalization point of $19 \mathrm{GeV}$. Here, we restrict ourselves to the $p=0$ case. The question to answer is how the spectral structures differ in contrast to the chiral case. We use the same temperature regime and discretization of the correlator data and leave the errors unchanged. The imaginary frequency dependence of $S_{4}$ in the finite mass case at vanishing spatial momentum is displayed in Fig. 13.

The results for the zero momentum spectral reconstructions at different temperature are given in Fig. 14, with the smooth BR method in the top panel and the conventional one in the bottom one. Qualitatively, the figures are very similar to the results in the chiral case. There exist two peaks, one at the origin and one above $1 \mathrm{GeV}$. The position of the second peak moves to higher frequencies as the temperature increases, while the height of the lowest lying peak decreases continuously. The only visible difference is that at low temperature the height of the peak around the origin is discernibly smaller than in the chiral case.

We again checked that the changes between the outcome at different temperatures are indeed attributable to in-medium effects by manually coarsening the $T=0.15 \mathrm{GeV}$ correlator data and repeating the reconstruction with it. The results of this procedure are similar to the ones in the quenched case: 

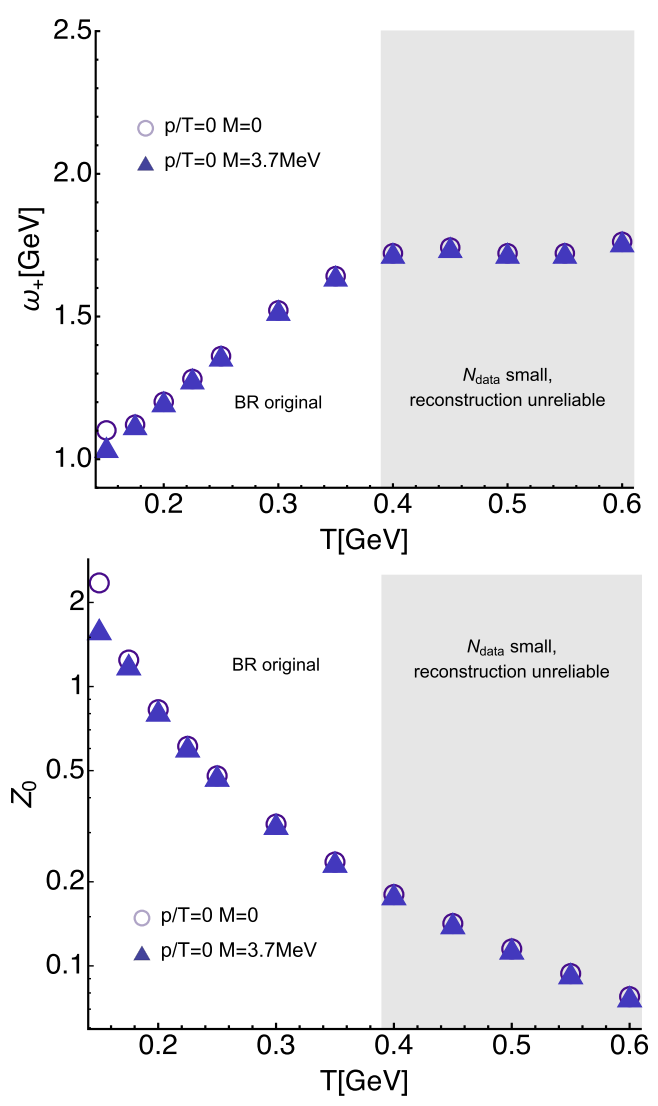

FIG. 15. (Top) position of the higher lying peak for different temperatures at vanishing momentum for the finite mass case (triangle) and the chiral case (circle). Except for the lowest $T$, no significant difference is observed. (Bottom) Amplitude of the lowest lying peak, which except for very low temperatures shows the same behavior between the chiral and finite mass cases.

The deletion of every second data point, i.e., $N_{\text {data }}=56$, weakens the peak strength while leaving the peak position unaffected. The reconstruction based on only every fourth data point corresponding to the situation at $T=0.6 \mathrm{GeV}$ fails to identify the encoded two-peak structure and is therefore deemed not trustworthy.

Just as in the chiral case, we determine the position of the second peak $\omega_{+}$and the height of the peak around the origin $Z_{0}$, shown in the top and bottom panels of Fig. 15 respectively. ${ }^{2}$ As is to be expected from the close resemblance of the reconstructed spectra, the values obtained do not differ markedly between the finite mass (triangle) and the chiral (circle) case. The only differences are found at low temperatures. The values of $\omega_{+}$for finite mass actually show a linear behavior down to $T=0.15 \mathrm{GeV}$, whereas in the chiral case, they deviate from a straight line at that

\footnotetext{
${ }^{2}$ Note that the quantity $\omega_{+}$shown here does not fully coincide with the conventional thermal mass at $|\boldsymbol{p}|=0$, as it is reconstructed from $S_{4}$, instead of $S_{4} \pm S_{s}$. Since the latter makes the spectral function nonsymmetric, we postpone its analysis to future work.
}

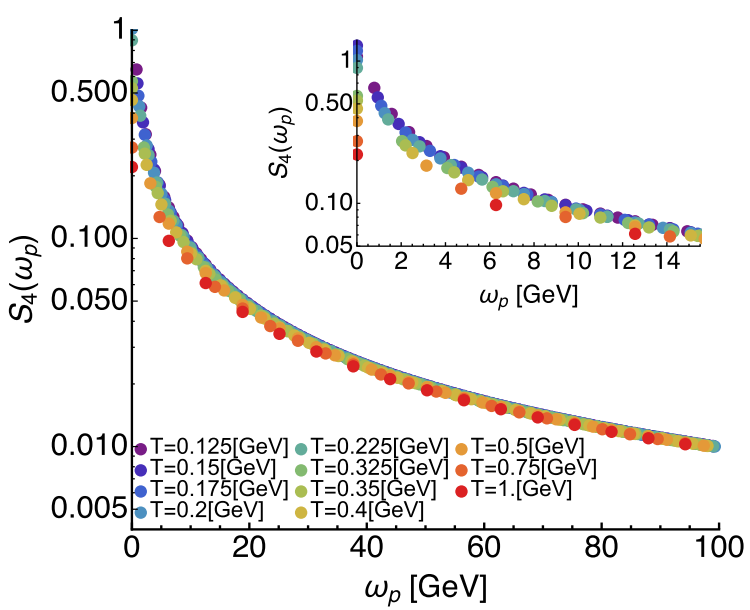

FIG. 16. Zero momentum imaginary frequency quark correlator at finite mass and finite temperature from the modern truncation with unquenched $N_{f}=2+1$ light quark flavors. Above $\omega_{p}>50 \mathrm{GeV}$, all correlators already lie very close to the $T=0$ curve. Numerical errors are smaller than the data-point markers.

point. $Z_{0}$ is smaller at low temperatures in the presence of a finite quark mass, but already at $T=0.2 \mathrm{GeV}$, no significant difference remains.

\section{B. Unquenched truncation with back-coupled $N_{f}=2+1$ quark flavors}

Our main result concerns the quark spectrum computed from the quark and gluon Dyson-Schwinger equations in a modern truncation incorporating $N_{f}=2+1$ light quark flavors with physical masses. In this case, we encounter a chiral crossover at a pseudocritical temperature of $T=$ $0.155 \mathrm{GeV}$ via the inflection point of the quark condensate and $T=0.160 \mathrm{GeV}$ via the chiral susceptibility in agreement with corresponding lattice results. We have computed $S_{4}$ along Matsubara frequencies at vanishing spatial momentum for 11 temperatures in a larger temperature range of $T \in[0.125,1.0] \mathrm{GeV}$, i.e., also for temperatures below the pseudocritical one, as shown in Fig. 16. At the same cutoff of $\Lambda=100 \mathrm{GeV}$ as before, this now corresponds to $N_{\text {data }} \in[127,16]$. The correlator computations have been checked to carry a numerical error of less than $\Delta D / D \leq 10^{-3}$, so that we can assign a corresponding diagonal correlation matrix to it. Both the smooth and original BR methods are carried out with a frequency discretization of $N_{\omega}=1000$ in the interval $\omega \in[0,20]$. The default model is set to a constant $m(\omega)=m_{0}$, and we carry out the reconstruction with the different choices $m_{0}=\{0.1,0.5,1.0,5.0,10\}$. The variance in the outcome is taken as the basis for the error bands depicted in the subsequently shown plots. In order to keep the presentation of the reconstructed spectra clear, we show in Fig. 17 only a subset of the reconstruction in a temperature range pertinent to the discussion below. For completeness, the full results are plotted in Appendix B in Fig. 23. 

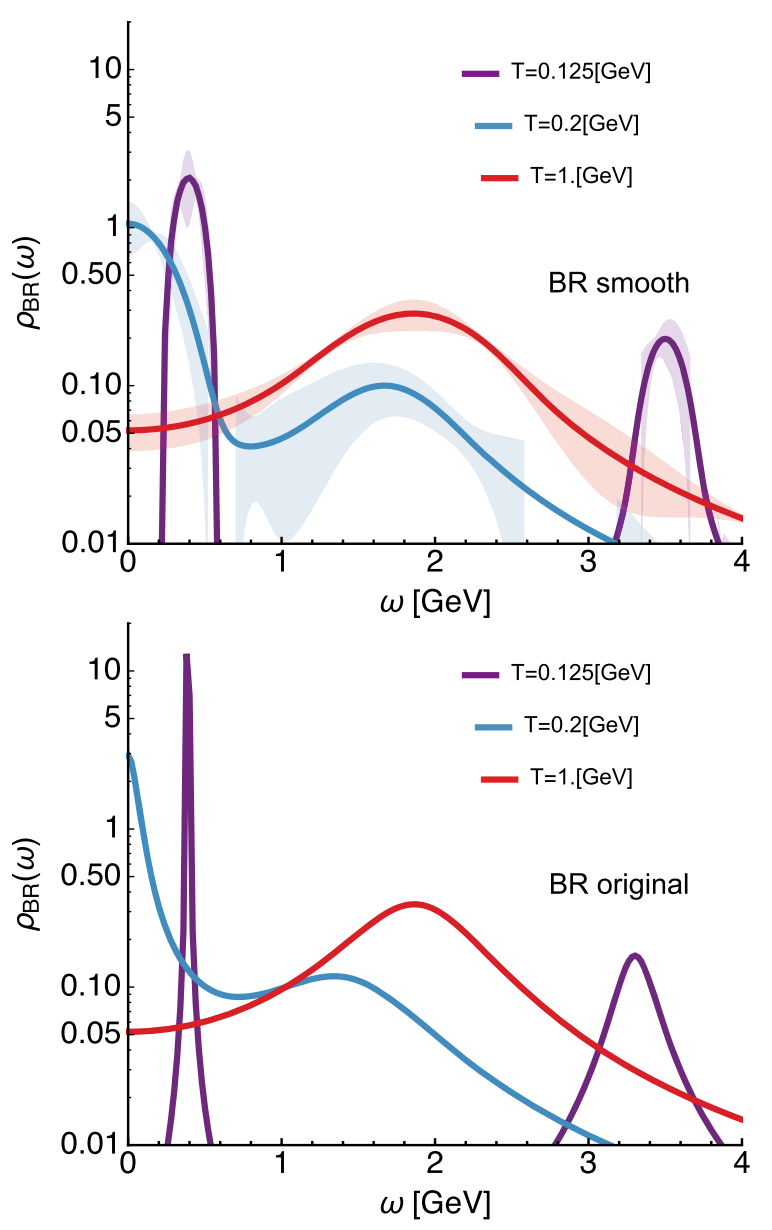

FIG. 17. Zero momentum reconstruction of the $T>0$ quark spectral function in the modern truncation with unquenched $N_{f}=2+1$ light quark flavors using the smooth BR (top) and the original BR (bottom) methods. For better readability, the plot contains only a subset of the reconstructed temperatures and is given in the logarithmic scale.

The first interesting result is that the reconstructions at low temperatures show unstable behavior that hints at a failure of the reconstruction limited to positive definite spectra. We see in Fig. 17 that at $T=0.125 \mathrm{GeV}$ two sharp peaks appear at positions very different from those at higher $T$. The results at and above the transition region, $T=0.150 \mathrm{GeV}$ and $T=0.175 \mathrm{GeV}$, appear to be in better shape at first glance. However, truncating these data sets (e.g., from $N_{\text {data }}=106$ to $N_{\text {data }}=94$ for the lowest temperature) actually changes the behavior of the spectral functions around $\omega=0$. The same tests for the two analyses in the previous subsections showed virtually no effect on the reconstruction, which is what is expected for a well-converged result. We thus conclude that in the truncation scheme with back-coupled quarks positivity violations characteristic for the low temperature spectral functions of the quarks persist for much larger temperatures than in the simple model case and prohibit convergence. Only the spectra at and above $T=0.2 \mathrm{GeV}$ do

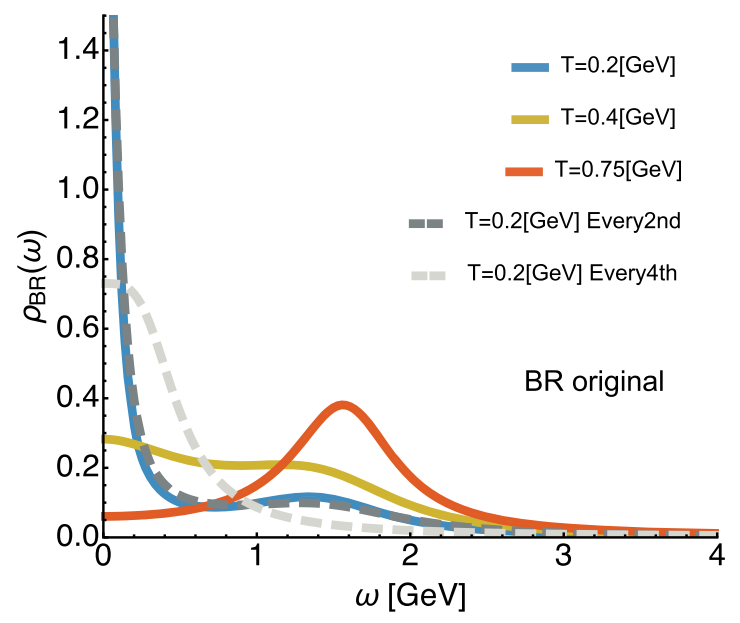

FIG. 18. Test of the reliability of the reconstruction at different temperatures based on the correlator made manually more sparse at $T=0.2 \mathrm{GeV}$. Using only every second data point of the original $N_{\text {data }}=80$ (dark grey) leaves the result virtually unchanged. When made more sparse to every fourth point, the Bayesian method only recovers the single peak at the origin (light grey). Nonetheless, clear in-medium modification at $T>0.2 \mathrm{GeV}$ is observed.

not show such artificial behavior and are therefore deemed trustworthy.

This is also indicated in Fig. 18, where we show the reconstruction outcome of taking the correlator at $T=$ $0.2 \mathrm{GeV}$ and $N_{\text {data }}=80$ and making it more sparse by a factor 2 (dark grey dashed) or factor 4 (light grey dashed). For $N_{\text {data }}=40$, the reconstruction is only very weakly affected, while for $N_{\text {data }}=20$, a sole peak at the origin remains. We conclude as before that the reconstruction eventually becomes unreliable at high temperature but that at intermediate $T$ we are able to observe genuine inmedium modification.

We now analyze the position of the peaks. Although we find the same number of peaks present as in the model calculation, their behavior under variations of temperature seems to be different. Whereas the amplitude of the lowest lying peak still decreases with increasing temperature, the position of the second peak does not show a clear pattern. In particular, it appears to not move monotonously to higher values of frequency with increasing temperature.

This is obvious from Fig. 19, where we plot again the location $\omega_{+}$of the second peak (upper panel) and the amplitude $Z_{0}$ of the zero mode (lower panel). For purpose of comparison, we also depict the results from the finite mass rainbow-ladder truncation as circles. Clear qualitative and quantitative differences are visible. Instead of monotonously rising in value, $\omega_{+}$appears to decrease first up to around $T=0.4 \mathrm{GeV}$ before then increasing again in an almost linear fashion toward higher temperatures. Interestingly, the initial downward trend starts in the low 

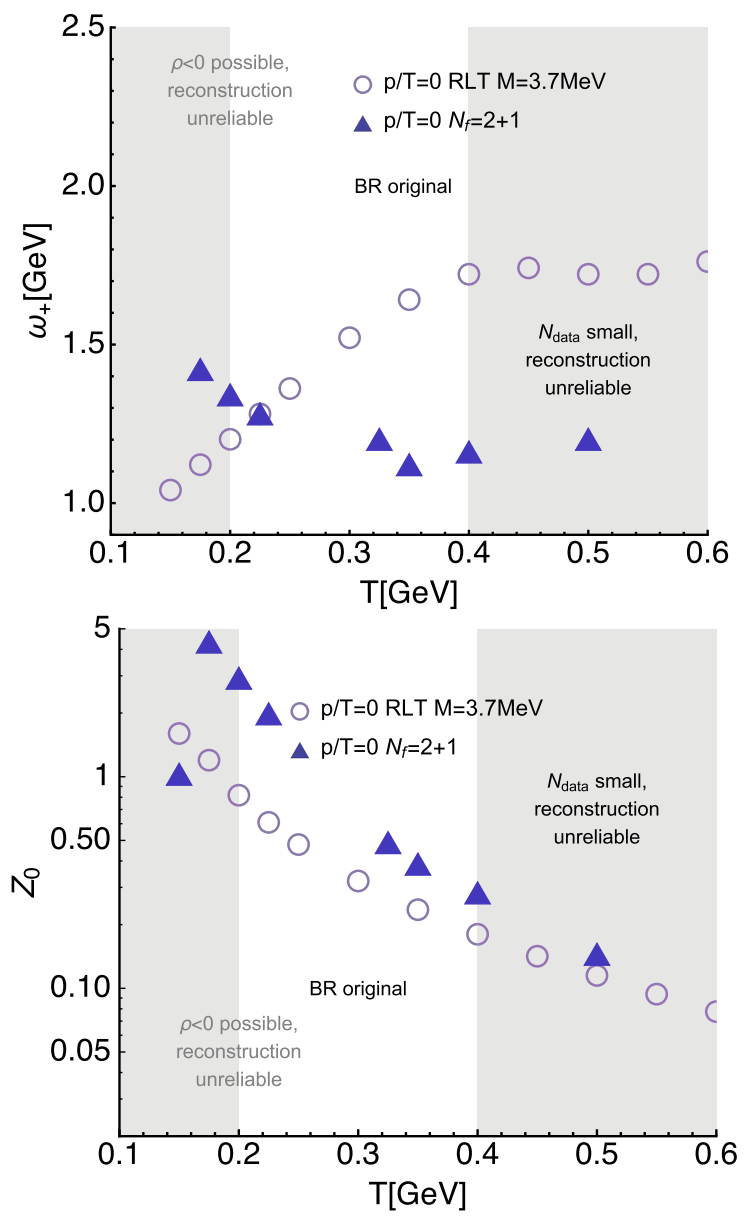

FIG. 19. (Top) position of the higher lying peak for different temperatures at vanishing momentum for the finite mass case (triangle) and the chiral case (circle). Except for the lowest $T$, no significant difference is observed. (Bottom) Amplitude of the lowest lying peak, which except for very low temperatures shows the same behavior between the chiral and finite mass cases.

temperature regime, where we did not deem the reconstruction reliable due to the possible presence of positivity violation. Then, we must further clarify whether the behavior of $\omega_{+}$up to $T=0.4 \mathrm{GeV}$ might still suffer from the influence of residual positivity violation. This will require the application of a reconstruction algorithm for general spectral functions, which is foreseen as the next step in this line of study.

$Z_{0}$ on the other hand behaves at least qualitatively similarly in the region where we trust the reconstruction. Below $T=0.15 \mathrm{GeV}$, it also shows a clear dip, which is related to the appearance of the artificial spiky structures at intermediate frequencies there. Above $T=0.2 \mathrm{GeV}$, it decreases monotonously.

Compared to the values reported in Ref. [14], the behavior of $\omega_{+}$here is quite similar. If the reconstructions between $T=0.2 \mathrm{GeV}$ and $T=0.4 \mathrm{GeV}$ are reliable, in particular, as they do not show any obvious pathologies, then we also observe a dip in $\omega_{+}$at intermediate temperatures. The height of the central peak, on the other hand, never fully vanishes in our case.

\section{CONCLUSIONS}

We have investigated the spectral properties of quarks in the Landau gauge, based on Dyson-Schwinger equations according to two different truncation schemes. In the rainbow-ladder approximation model, both the chiral and finite current quark mass cases have been considered, while our main result concerns quark spectra in a modern truncation with $N_{f}=2+1$ unquenched flavors of light medium quarks.

The reconstruction of the spectral functions was based on a recently developed Bayesian approach, the so-called BR method, formulated in imaginary frequencies. We further developed in this study a low-gain variant of the BR method, which successfully suppresses numerical ringing, which can affect the original BR method and in turn helps us to unambiguously determine the number of physical peaks in the spectrum. The accuracy of the reconstruction further benefits from the use of the KällenLehmann kernel instead of the Euclidean one.

In mock data tests, we have shown the capabilities and limitations of our Bayesian reconstruction approach for both a best-case scenario with correlator precision $\Delta D / D=10^{-8}$ and a real-world setting with $\Delta D / D=$ $10^{-3}$. In the case with two peaks, which is most relevant for our study, the combination of the conventional and smooth BR methods allowed us to unambiguously identify the number of encoded peaks, while an estimate of the peak properties, e.g., their position, was achieved within 15\%$20 \%$ of the correct value. On the other hand, in the most challenging but least likely realized case, where two rather broad peaks at high frequency were located close to each other, it required more than the best-case scenario to infer the presence of all features.

The reconstructed spectra for the rainbow-ladder truncation model with vanishing and finite current quark mass showed very similar behavior. At low temperatures, two peaks were present, one at the frequency origin and another one above $\omega=1 \mathrm{GeV}$. Changing the temperature or changing the spatial momenta induced qualitatively similar changes. The lowest lying peak height diminished but did not vanish up to the highest parameter values investigated. The second peak both broadened and moved to higher frequencies.

A quantitative analysis of the height of the low lying peak $Z_{0}$ and position of the second peak $\omega_{+}$revealed a different behavior than reported in previous studies. We did not find any indication of a nonmonotonicity in $\omega_{+}$ with respect to temperature, and our value for $Z_{0}$ always took on finite values in contrast to a vanishing $\omega=0$ peak in Ref. [14].

We have made sure that the observed changes in $Z_{0}$ and $\omega_{+}$with temperature can be attributed to the thermal 
medium. To disentangle the effects from a degrading of the reconstruction due to fewer available data points at high temperature, we repeated the reconstructions with correlator data sets manually made more sparse and identified the regime where the Bayesian method is reliable. And indeed, in the region where the reconstruction can be trusted, we found that $\omega_{+}$shows a linear rise with $T$ qualitatively compatible with hard-thermal loop predictions. At the same point where the reconstruction became unreliable, we also saw that the linear rise began to artificially flatten off.

In the unquenched truncation scheme with $N_{f}=2+1$ flavors of light medium quarks, the positive definite Bayesian approach was challenged at low and high temperatures. For $T<0.2 \mathrm{GeV}$, we found indications that nonpositive spectral contributions were present, which led to artificially spiky structures. On the other hand a test with input data, manually made more sparse, showed that for $N_{\text {data }}<20$ the reconstruction also became unreliable. In the intermediate temperature window, we observed again twopeak structures with the lower one decreasing monotonously in height. The second peak, however, behaved very differently than before as it then appeared to exhibit a dip in $\omega_{+}$, similar to the behavior reported in Ref. [14].

In order to unambiguously determine whether the nonmonotonous behavior of $\omega_{+}$can be attributed to physics encoded in the correlator, we will have to extend the analysis of this study in the future to nonpositive spectral functions. In the context of gluon spectral functions in lattice QCD, a generalization of the BR method has been proven a useful tool [19]. Implementing a smooth version of this generalized BR method will constitute an important step toward a robust and quantitative picture of the low

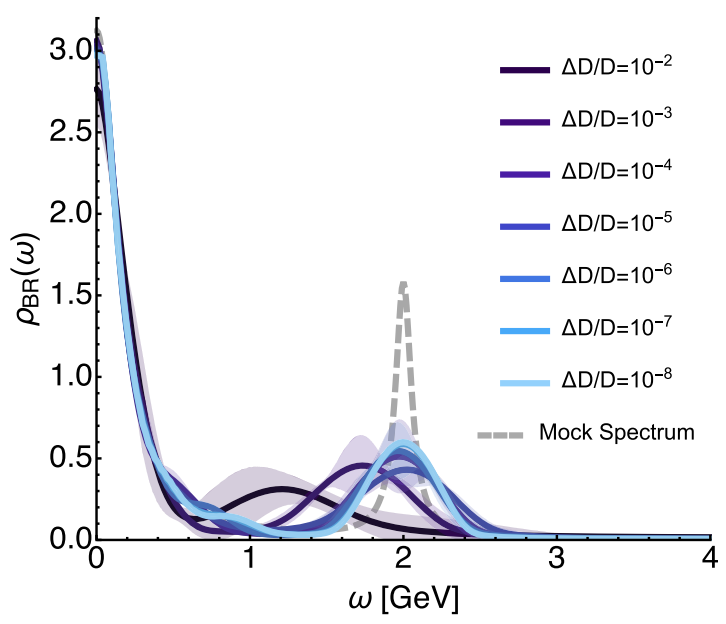

temperature regime of quark spectra, which is work in progress.

\section{ACKNOWLEDGMENTS}

C. F. and C. W. were supported by the German Federal Ministry of Education and Research under Contract No. 05P15RGFCA and the Helmholtz International Center for the Facility for Antiproton and Ion Research (FAIR) within the Landes-Offensive zur Entwicklung Wissenschaftlich-ökonomischer Exzellenz (LOEWE) of the State of Hesse. J. P. and A. R. acknowledge support by the ExtreMe Matter Institute, Grants No. ERC-AdG290623 and No. BMBF 05P12VHCTG, as well as that this work is part of and supported by the DFG Collaborative Research Centre "SFB 1225 (ISOQUANT)."

\section{APPENDIX A: MOCK TEST BAYESIAN CONTINUUM LIMIT}

In this Appendix, we present figures for the explicit approach of the mock spectral reconstructions of Sec. III B toward the Bayesian continuum limit at fixed $N_{\text {data }}=128$. Each of the figures contains seven solid curves, denoting the reconstruction according to an assigned relative error $\Delta D / D=\left[10^{-8}, 10^{-2}\right]$. In the upper panel, the smooth BR is deployed, while in the lower one, it is the original BR method. The darkest curve corresponds to the largest error. In Fig. 20, the two-peak scenario is shown, while Fig. 21 contains the results for three-peak scenario with the second peak lying close to that at the origin. Figure 22 shows the outcome for a three-peak scenario with two closely placed structures at finite $\omega$.

FIG. 20. Mock data analysis of reconstruction reliability for a two-peak scenario (grey dashed). Both the smooth BR (left) as well as the original BR (right) methods are shown. While the latter unambiguously shows only two features and is devoid of ringing, it approaches the Bayesian continuum limit more slowly than the original BR. 

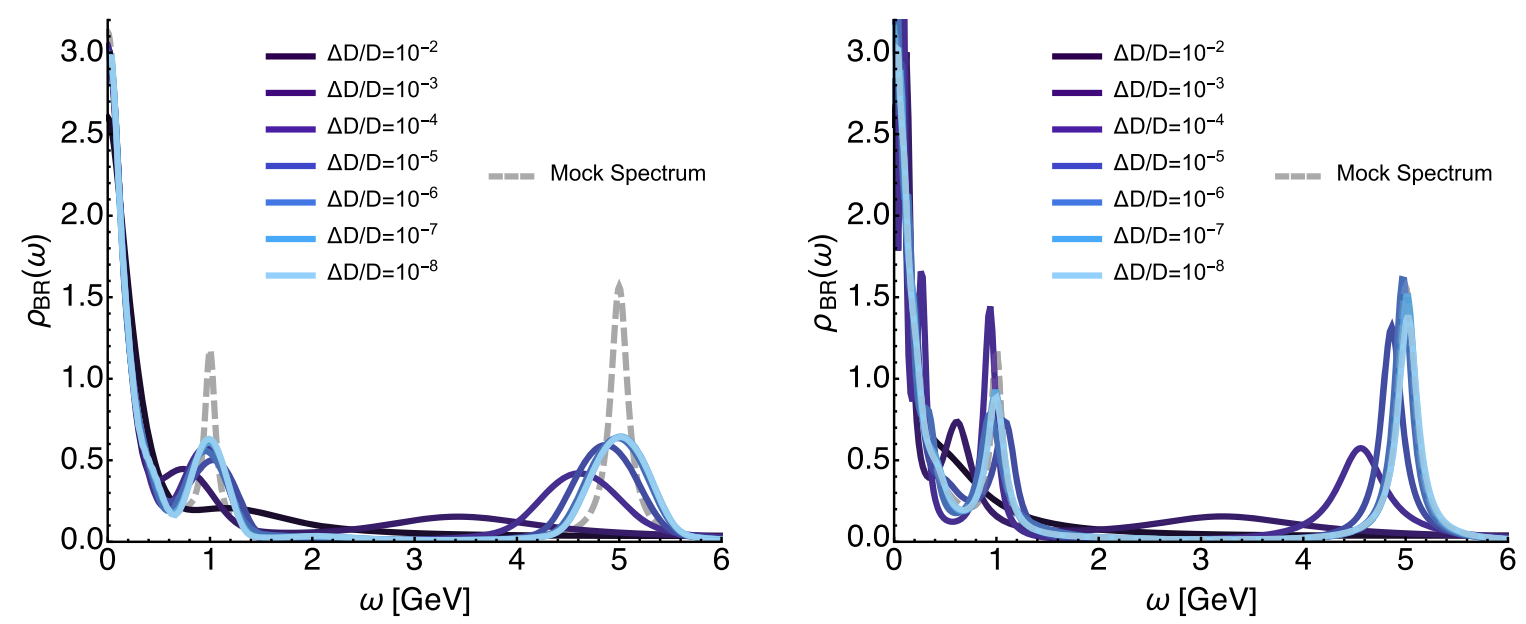

FIG. 21. Mock data analysis of reconstruction reliability for a three-peak scenario (grey dashed) with two peaks closely positioned off the origin. Both the smooth BR (left) as well as the original BR (right) methods are shown.
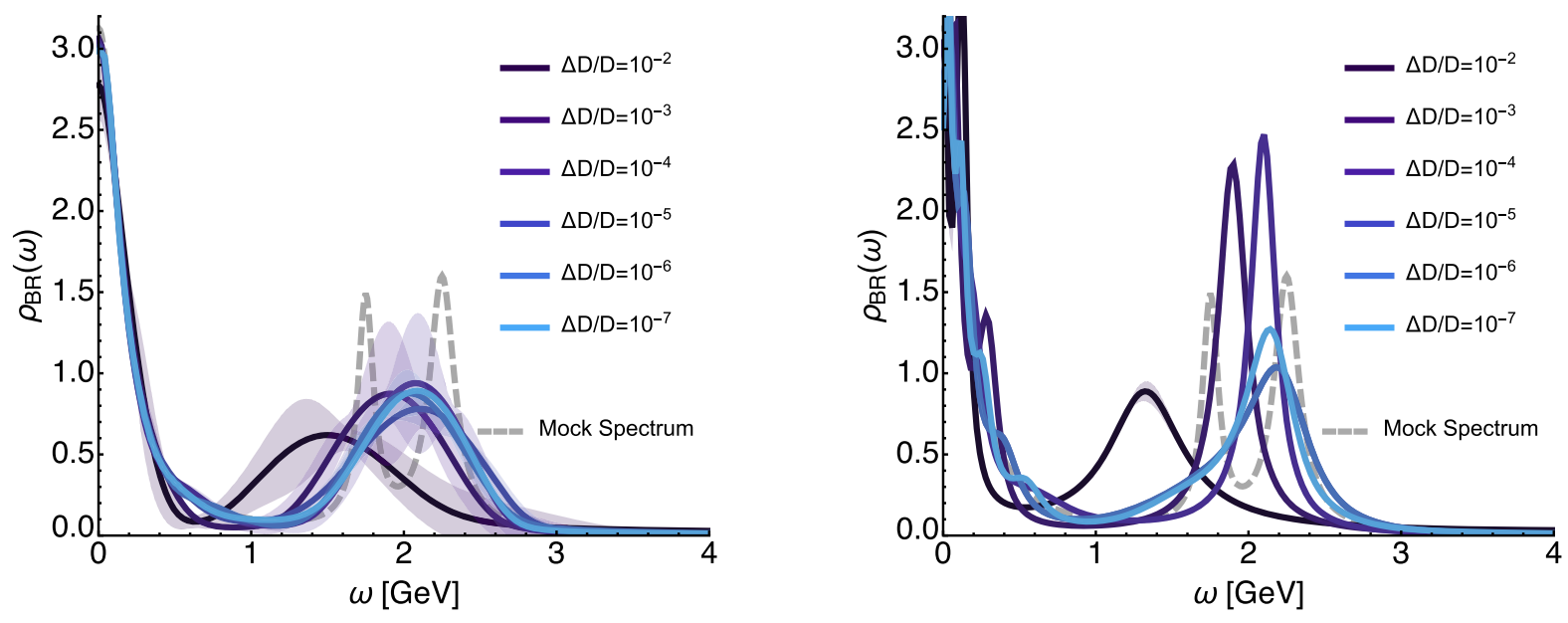

FIG. 22. Mock data analysis of reconstruction reliability for a three-peak scenario (grey dashed) with two peaks closely positioned off the origin. Both the smooth BR (left) as well as the original BR (right) methods are shown.

\section{APPENDIX B: COMPLETE SPECTRAL RECONSTRUCTIONS FOR THE UNQUENCHED CASE}

Here, we plot in Fig. 23 for completeness the spectral reconstructions for the unquenched truncation with back-coupled $N_{f}=2+1$ quark flavors. One observes the appearance of artificial peaked structures at the lowest temperature $T=0.125 \mathrm{GeV}$, while at $T=0.2 \mathrm{GeV}$ and above, we obtain the same number of peaks as in the model computations. As was discussed in the context of Fig. 19, the position of the peak located at finite frequencies, however, displays a qualitatively different behavior here than in the model truncation. 

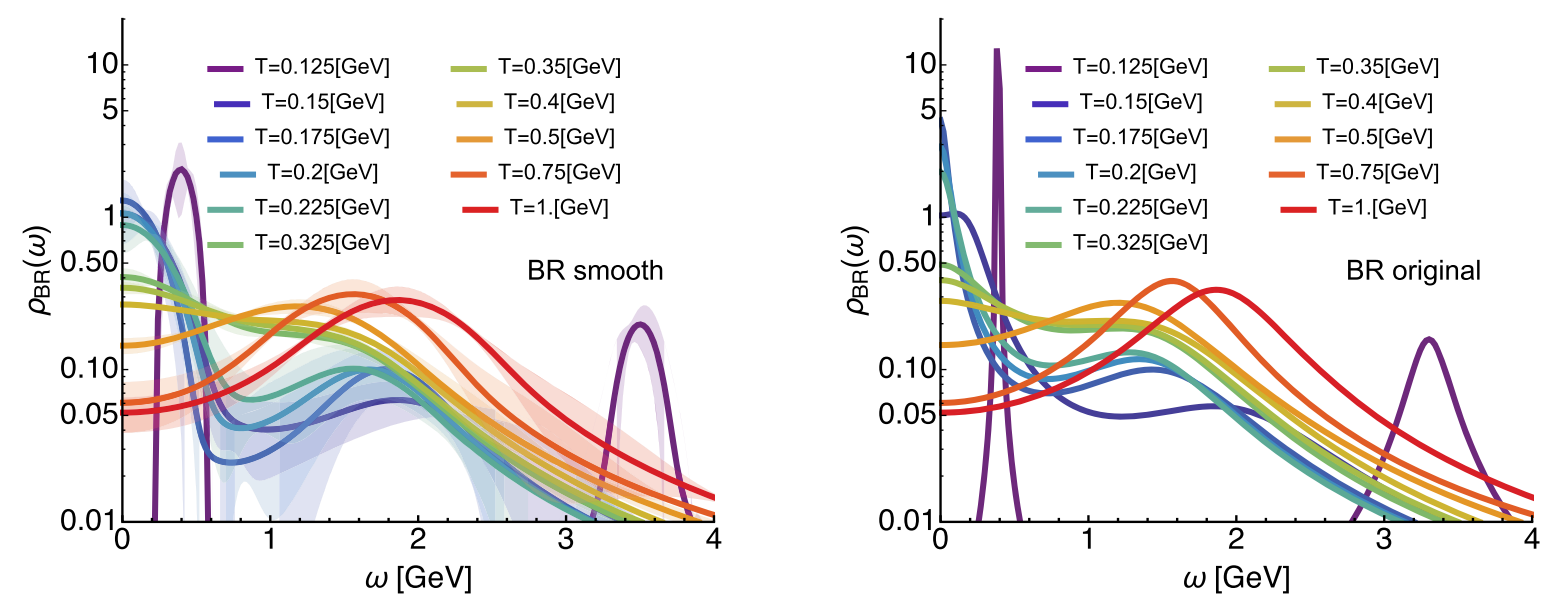

FIG. 23. Zero momentum reconstruction of the $T>0$ quark spectral function in the modern truncation with unquenched $N_{f}=2+1$ light quark flavors using the smooth BR (top) and the original BR (bottom) methods. For better readability, the plot is given in the logarithmic scale.

\section{APPENDIX C: TEST OF THE EVIDENCE PROBABILITY COMPUTATION}

In contrast to the BR method, where the hyperparameter $\alpha$ is self-consistently integrated out a priori, MEM-like approaches marginalize $\alpha$ at the end of the reconstruction procedure [35,37]; i.e., in MEM, one conventionally computes the corresponding spectrum $\rho_{\alpha}$ for many different values of $\alpha$ and then determines the probability distribution $P[\alpha \mid \rho, D, I]$. The individual $\rho_{\alpha}$ are subsequently averaged, weighted by $P[\alpha \mid \rho, D, I]$. In order to compute $P[\alpha \mid \rho, D, I]$, one, however, relies on both the assumptions that the posterior probability is highly peaked and that it allows for a Gaussian approximation. Neither is tested in practice.

Common lore states that $P[\alpha \mid \rho, D, I]$ will have a peak at a finite $\alpha$ for which one particular spectrum contributes most strongly. If the maximum were at $\alpha=0$, the method reverts to an underdetermined $\chi^{2}$ fit, and no unique extremum exists. Here, we give numerical evidence that the existence of a peak in the approximated $P[\alpha \mid \rho, D, I]$ depends on the choice of search space used. Furthermore, if the search space is extended to the full size of the problem (in which there still exists a unique Bayesian answer), we find that only a maximum at $\alpha=0$ remains.

We use the same mock data as in the two-peak scenario in Sec. III B and compare four different scenarios. We deploy the MEM with limited search space $S_{\text {BR }}$ and $N_{\text {base }}=$ $N_{\text {data }}$ according to Bryan and compare with (solid line) an implementation without restriction, where $N_{\text {base }}=N_{\omega}$ (dashed line). The Bayesian result is selected with a step tolerance in the minimizer of $\Delta=5 \times 10^{-8}$. In addition, we replace the Shannon-Jaynes entropy by the BR prior and repeat the reconstruction with $S_{\mathrm{BR}}$ and restricted search space $N_{\text {base }}=N_{\text {data }}$ (solid line) or without, i.e., using $S_{\mathrm{BR}}$ and $N_{\text {base }}=N_{\omega}$ (dashed line). We have of course adapted the computation of $P[\alpha \mid \rho, D, I]$ to this new prior. The results for the probabilities are shown in Fig. 24.

We find indeed that only for the restricted search space a peak at finite values of $\alpha$ remains. This issue is independent of the actual regulator used; both $S_{\mathrm{SJ}}$ and $S_{\mathrm{BR}}$ show the same trend. We believe that the underlying reason is that in the presence of a restricted search space the minimizer is at some point not able to lower the value of $L$, while in the full search space, it can be brought very close to zero. This finite minimal value of $L$ then prohibits the probability of rising further. Since the Bayesian answer is unique if it exists [37], we conclude that it is the approximations made to determine $P[\alpha \mid \rho, D, I]$ which prevent us from obtaining that unique result in the full search space.

As a consequence, we revert to the historic MEM choice of setting $\alpha$ such that $L=N_{\text {data }}$ in case of the smooth BR method, where an a priori marginalization of the hyperparameter is not analytically feasible.

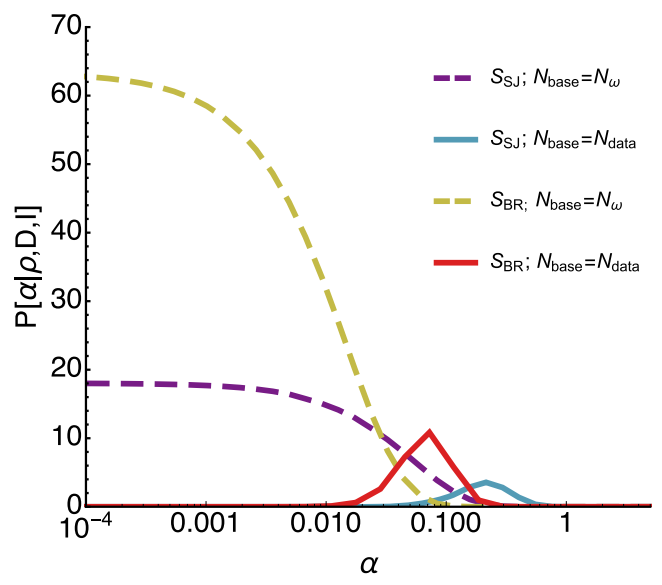

FIG. 24. Approximate probability distribution for the hyperparameter $P[\alpha \mid \rho, D, I]$ computed according to four different scenarios. We compare the use of the Shannon-Jaynes entropy $S_{\mathrm{BR}}$ with Bryan's search space $N_{\text {base }}=N_{\text {data }}$ (turquoise solid) or the full search space $N_{\text {base }}=N_{\omega}$ (blue dashed). The other two curves denote the BR prior $S_{\mathrm{BR}}$ with Bryan's search space $N_{\text {base }}=N_{\text {data }}$ (red solid) or full search space $N_{\text {base }}=N_{\omega}$ (yellow dashed). Only by artificially restricting the search space do we find a maximum at finite $\alpha$. 
[1] B. Muller and J. L. Nagle, Annu. Rev. Nucl. Part. Sci. 56, 93 (2006).

[2] E. Shuryak, Prog. Part. Nucl. Phys. 62, 48 (2009).

[3] P. Braun-Munzinger and J. Wambach, Rev. Mod. Phys. 81, 1031 (2009).

[4] A. Andronic, Int. J. Mod. Phys. A 29, 1430047 (2014).

[5] P. Foka and M. A. Janik, Rev. Phys. 1, 172 (2016).

[6] J. A. Mueller, C. S. Fischer, and D. Nickel, Eur. Phys. J. C 70, 1037 (2010).

[7] D. Nickel, Ann. Phys. (Amsterdam) 322, 1949 (2007).

[8] M. Harada, Y. Nemoto, and S. Yoshimoto, Prog. Theor. Phys. 119, 117 (2008).

[9] M. Harada and S. Yoshimoto, arXiv:0903.5495.

[10] F. Karsch and M. Kitazawa, Phys. Lett. B 658, 45 (2007).

[11] F. Karsch and M. Kitazawa, Phys. Rev. D 80, 056001 (2009).

[12] S.-x. Qin, L. Chang, Y.-x. Liu, and C. D. Roberts, Phys. Rev. D 84, 014017 (2011).

[13] S.-x. Qin and D. H. Rischke, Phys. Rev. D 88, 056007 (2013).

[14] F. Gao, S.-X. Qin, Y.-X. Liu, C. D. Roberts, and S. M. Schmidt, Phys. Rev. D 89, 076009 (2014).

[15] S. Strauss, C. S. Fischer, and C. Kellermann, Phys. Rev. Lett. 109, 252001 (2012).

[16] M. Haas, L. Fister, and J. M. Pawlowski, Phys. Rev. D 90, 091501 (2014).

[17] N. Christiansen, M. Haas, J. M. Pawlowski, and N. Strodthoff, Phys. Rev. Lett. 115, 112002 (2015).

[18] D. Dudal, O. Oliveira, and P. J. Silva, Phys. Rev. D 89, 014010 (2014).

[19] E.-M. Ilgenfritz, J. M. Pawlowski, A. Rothkopf, and A. Trunin, Eur. J. Phys. C 78, 127 (2018).

[20] E. Braaten, R. D. Pisarski, and T.-C. Yuan, Phys. Rev. Lett. 64, 2242 (1990).

[21] A. Peshier and M. H. Thoma, Phys. Rev. Lett. 84, 841 (2000).

[22] P. B. Arnold, G. D. Moore, and L. G. Yaffe, J. High Energy Phys. 06 (2002) 030.

[23] T. Kim, M. Asakawa, and M. Kitazawa, Phys. Rev. D 92, 114014 (2015).

[24] S. Y. F. Liu and R. Rapp, arXiv:1612.09138.

[25] E. Braaten and R. D. Pisarski, Nucl. Phys. B337, 569 (1990).

[26] G. Baym, J.-P. Blaizot, and B. Svetitsky, Phys. Rev. D 46, 4043 (1992).

[27] J.-P. Blaizot and J.-Y. Ollitrault, Phys. Rev. D 48, 1390 (1993).

[28] A. Schaefer and M. H. Thoma, Phys. Lett. B 451, 195 (1999).
[29] M. Kitazawa, T. Kunihiro, and Y. Nemoto, Phys. Lett. B 633, 269 (2006).

[30] M. Kitazawa, T. Kunihiro, and Y. Nemoto, Prog. Theor. Phys. 117, 103 (2007).

[31] K. Kamikado, N. Strodthoff, L. von Smekal, and J. Wambach, Eur. Phys. J. C 74, 2806 (2014).

[32] R.-A. Tripolt, L. von Smekal, and J. Wambach, Phys. Rev. D 90, 074031 (2014).

[33] J. M. Pawlowski and N. Strodthoff, Phys. Rev. D 92, 094009 (2015).

[34] N. Strodthoff, Phys. Rev. D 95, 076002 (2017).

[35] M. Jarrell and J. Gubernatis, Phys. Rep. 269, 133 (1996).

[36] W. H. Press, S. A. Teukolsy, W. T. Vetterling., and B. P. Flannery, Numerical Recipes 3rd Edition: The Art of Scientific Computing (Cambridge University Press, New York, USA, 2007).

[37] M. Asakawa, T. Hatsuda, and Y. Nakahara, Prog. Part. Nucl. Phys. 46, 459 (2001).

[38] Y. Burnier and A. Rothkopf, Phys. Rev. Lett. 111, 182003 (2013).

[39] A. Rothkopf, Phys. Rev. D 95, 056016 (2017).

[40] S. Kim, P. Petreczky, and A. Rothkopf, Phys. Rev. D 91, 054511 (2015).

[41] C. S. Fischer and J. Luecker, Phys. Lett. B 718, 1036 (2013).

[42] R. Aouane, F. Burger, E. M. Ilgenfritz, M. Mller-Preussker, and A. Sternbeck, Phys. Rev. D 87, 114502 (2013).

[43] S. Borsanyi, Z. Fodor, C. Hoelbling, S. D. Katz, S. Krieg, C. Ratti, and K. K. Szabo (Wuppertal-Budapest Collaboration), J. High Energy Phys. 09 (2010) 073.

[44] C. S. Fischer, L. Fister, J. Luecker, and J. M. Pawlowski, Phys. Lett. B 732, 273 (2014).

[45] C. S. Fischer, J. Luecker, and C. A. Welzbacher, Phys. Rev. D 90, 034022 (2014).

[46] G. Eichmann, C. S. Fischer, and C. A. Welzbacher, Phys. Rev. D 93, 034013 (2016).

[47] C. S. Fischer, Phys. Rev. Lett. 103, 052003 (2009).

[48] C. S. Fischer, A. Maas, and J. A. Muller, Eur. Phys. J. C 68, 165 (2010).

[49] A. Maas, J. M. Pawlowski, L. von Smekal, and D. Spielmann, Phys. Rev. D 85, 034037 (2012).

[50] J. S. Ball and T.-W. Chiu, Phys. Rev. D 22, 2542 (1980).

[51] J. Pawlowski and A. Rothkopf, Phys. Lett. B 778, 221 (2018).

[52] C. A. Welzbacher, Ph.D. thesis, Justus-Liebig-Universität, 2016.

[53] A. Rothkopf, J. Comput. Phys. 238, 106 (2013).

[54] H. T. Ding, A. Francis, O. Kaczmarek, F. Karsch, H. Satz, and W. Soeldner, Phys. Rev. D 86, 014509 (2012). 